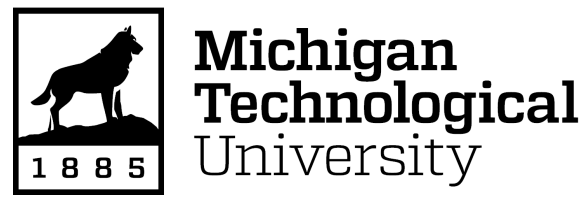

Michigan Technological University Digital Commons @ Michigan Tech

Dissertations, Master's Theses and Master's Reports

2021

\title{
AUXIN-INDUCED DEGRADATION OF DREAM PROTEINS, LIN-9 AND LIN-54, IN CAENORHABDITIS ELEGANS
}

Karli E. Chosa

Michigan Technological University, kechosa@mtu.edu

Copyright 2021 Karli E. Chosa

\section{Recommended Citation}

Chosa, Karli E., "AUXIN-INDUCED DEGRADATION OF DREAM PROTEINS, LIN-9 AND LIN-54, IN CAENORHABDITIS ELEGANS", Open Access Master's Thesis, Michigan Technological University, 2021.

https://doi.org/10.37099/mtu.dc.etdr/1159

Follow this and additional works at: https://digitalcommons.mtu.edu/etdr

Part of the Molecular Biology Commons 


\title{
AUXIN-INDUCED DEGRADATION OF DREAM PROTEINS, LIN-9 AND LIN-54, IN CAENORHABDITIS ELEGANS
}

\author{
By \\ Karli Elizabeth Chosa
}

\begin{abstract}
A THESIS
Submitted in partial fulfillment of the requirements for the degree of MASTER OF SCIENCE In Biological Sciences
\end{abstract}

MICHIGAN TECHNOLOGICAL UNIVERSITY 2021

(C) 2021 Karli E. Chosa 
This thesis has been approved in partial fulfillment of the requirements for the Degree of MASTER OF SCIENCE in Biological Sciences.

Department of Biological Sciences

Thesis Advisor: $\quad$ Paul D. Goetsch

Committee Member: Xiaohu Tang

Committee Member: Stephen Techtmann

Department Chair: Chandrashekhar P. Joshi 


\section{Table of Contents}

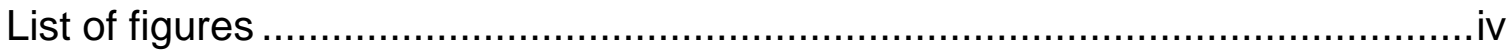

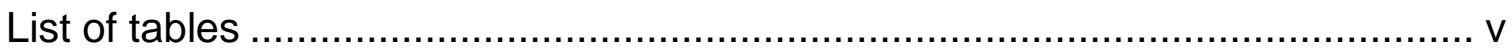

Acknowledgements …........................................................................

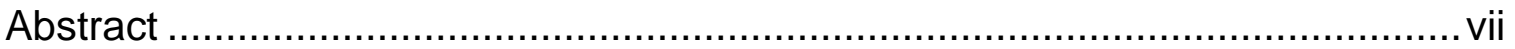

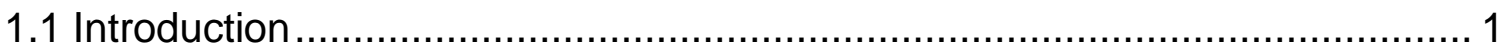

1.1.2 The Auxin-Inducible Degron (AID) System ............................ 2

1.1.3 DREAM Complex .............................................................. 4

1.1.4 Dysfunction of MuvB and DREAM ..................................... 5

1.1.5 Study Hypothesis ........................................................ 8

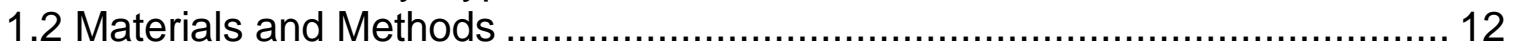

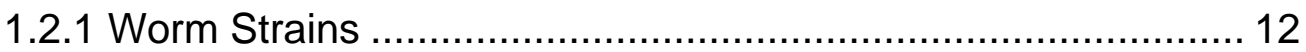

1.2.2 Liquid Worm Growth ............................................................ 12

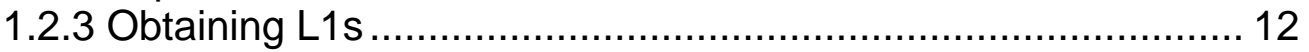

1.2.4 Auxin Treatment Time Course ......................................... 13

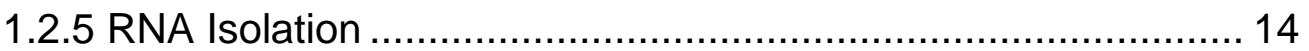

1.2.6 Reverse Transcription ......................................................... 15

1.2.7 Quantitative PCR ........................................................... 15

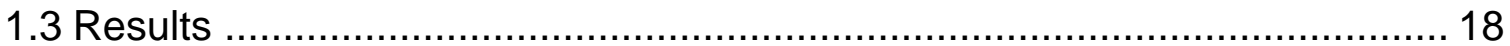

1.3.1 Auxin-induced degradation of LIN-54 does not affect expression

of house-keeping genes ........................................................................ 18

1.3.2 Auxin-induced degradation of LIN-54 results in activation of

DREAM target genes.

1.3.3 Auxin-induced degradation of LIN-9 does not affect expression of house-keeping genes ..................................................................... 19

1.3.4 Auxin-induced degradation does not cause significant effect on

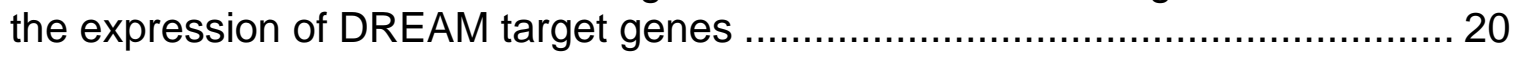

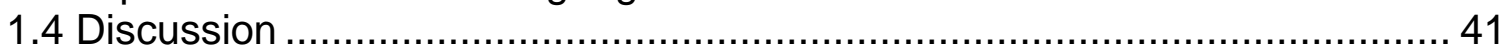

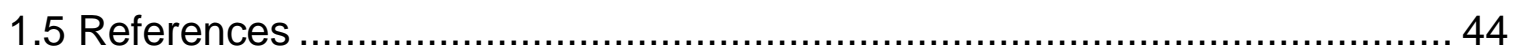




\section{List of figures}

Figure 1. The auxin inducible degron (AID) system .................................... 10

Figure 2. C.elegans DRM complex......................................................... 11

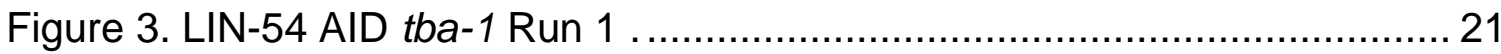

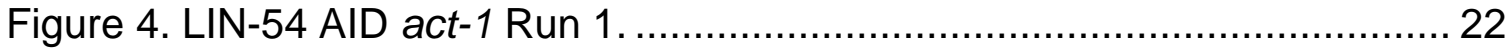

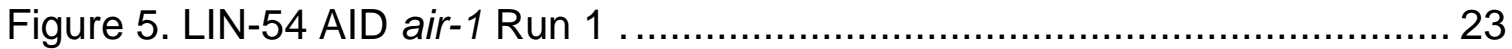

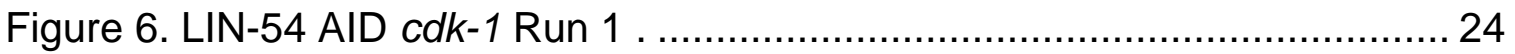

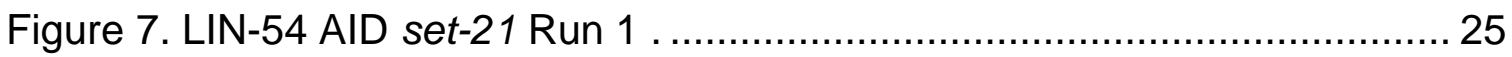

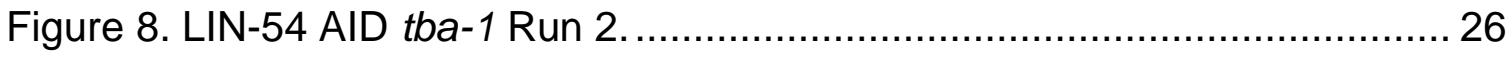

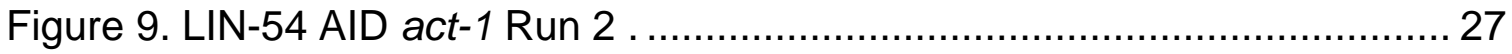

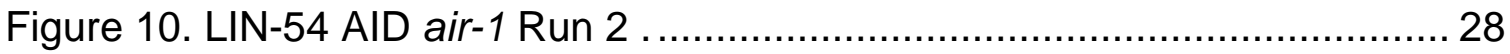

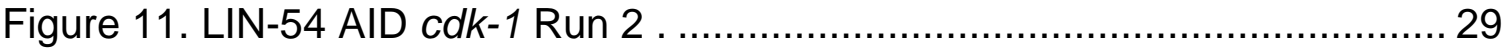

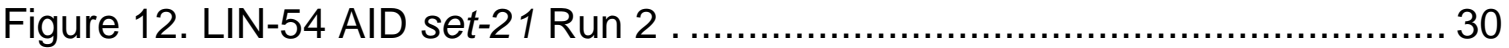

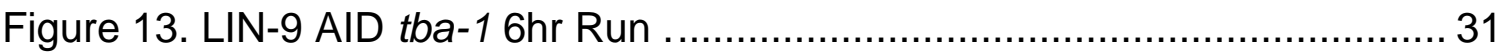

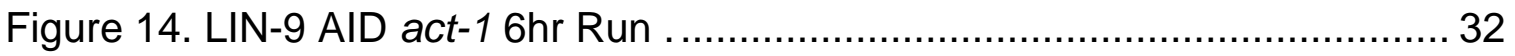

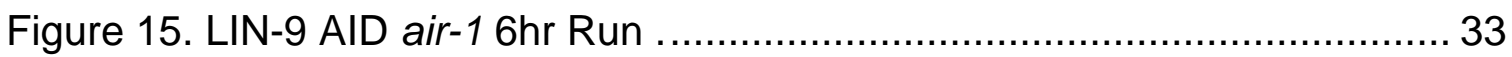

Figure 16. LIN-9 AID cdk-1 6hr Run ....................................................... 34

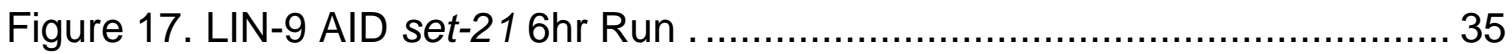

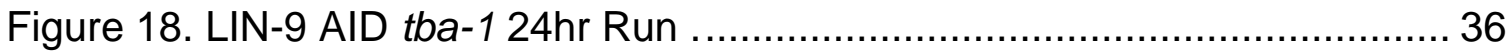

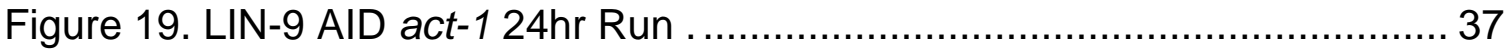

Figure 20. LIN-9 AID air-1 24hr Run .................................................... 38

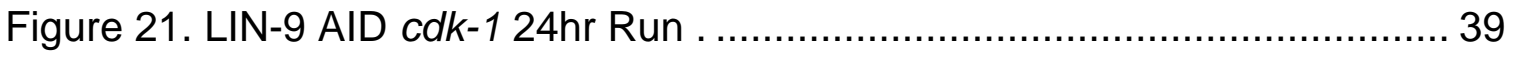

Figure 22. LIN-9 AID set-21 24hr Run .................................................... 40 


\section{List of tables}

Table 1. Primers and their corresponding DNA sequences............................ 17 


\section{Acknowledgements}

I would like to express my deepest gratitude to my advisor, Dr. Paul Goetsch. Thank you for guidance, expertise, and allowing me to work on this project. I have learned a great deal being a part of your lab, thank you for providing me with this opportunity.

Thank you to my committee members, Dr. Xiaohu Tang and Dr. Stephen Techtmann. I greatly appreciate you volunteering your time and knowledge to this project.

To my family and friends, without your unwavering support this would have never been possible. Acacia, our weekly humane society visits provided some muchneeded stress relief, thank you for always being willing to listen on our dog walking adventures. Mom, I cannot truly express in words what you have done and continue to do for me, you will always be my rock. 


\section{ABSTRACT}

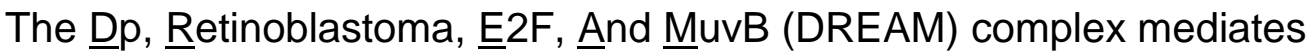
transcriptional repression and is highly conserved throughout a number of species, including vertebrates, Drosophila melanogaster, and Caenorhabditis elegans. Differing from mammalian DREAM, C.elegans DRM, appears to act solely in a repressive role, with the MuvB subcomplex (LIN-9, LIN-37, LIN-52, LIN-53, and LIN-54) playing a key role in the repression of genes. In this study, we use the auxin-inducible degron (AID) system, an effective, fast-acting, tool used in the degradation of degron-tagged proteins to individually deplete two key proteins of the MuvB subcomplex, LIN-9 and LIN-54, in C. elegans. The AID system relies on the expression of the F-box protein, transport inhibitor response 1 (TIR1), which in the presence of auxin acts as the substrate recognition component for the SKP1-CUL1-F-box (SCF) E3 ubiquitin ligase complex, leading to the degradation of tagged proteins. In degron-tagged LIN-54 worms, using a 6hour auxin time course, we observed that DREAM target genes become significantly upregulated. Expression of DREAM target genes increased with longer exposure to auxin, indicating that LIN-54 plays a key role in the regulation of DREAM target genes. However, in both a 6-hour and 24-hour auxin time course experience, degron-tagged LIN-9 worms showed no uniform nor significant upregulation of DREAM target genes compared to ethanol vehicle control. These results demonstrate that LIN-54, the sole DNA-binding protein of MuvB, plays a more important role in MuvB's repression of genes than the core protein of MuvB, LIN-9. We recommend further study into these two proteins 
using the AID system to further explore their roles in MuvB and DREAM complex function. 


\subsection{INTRODUCTION}

The expanding experimental capability to manipulate protein levels in both cells and living organisms proves to be instrumental in our understanding of what role these proteins play in biological processes. Traditional methods of protein manipulation, such as gene knockout and RNA interference (RNAi), are widely used to examine the effects of specific protein loss in an organism or cells. However, these techniques are indirect, dependent on protein stability, and not easily reversible [1]. One new method to manipulate protein levels in cells and organisms, the auxin-inducible degron (AID) system, is effective in its depletion of proteins and is conditional, fast-acting, and reversible [1, 2, 3, 4]. This system relies on both the presence of auxin and the expression of Arabidopsis thaliana F-box protein TIR1, which serves as the substrate recognition component for the SCF E3 ubiquitin ligase complex, resulting in the degradation of degron tagged proteins by a proteosome [1, 2]. We seek to use this system to study DREAM complex function in Caenorhabditis elegans. The DREAM complex is a transcriptional repressor that is highly conserved across numerous species, with MuvB acting as the mediator of repression in the C. elegans DREAM complex, known as DRM $[5,6]$. MuvB subunits LIN-54 and LIN-9 were individually tagged with the degron tag coupled to green fluorescent protein. We believe that the degradation of LIN-54 and/or LIN-9 will result in the activation of DREAM target genes that would otherwise be repressed. 


\subsubsection{The Auxin-Inducible Degron (AID) System}

The AID system relies on the use of a small amino acid sequence, also called a degron, that acts as a target for the SKP1, CUL1, F-box (SCF) E3 ubiquitin ligase complex [1, 2]. In the ubiquitin pathway, chains of ubiquitin attach to a target substrate, in this case the degron, which allows the modified protein to be recognized and degraded by the proteosome [7]. Cul1 acts as the major scaffold and brings together Skp1 and RBX1, an E3 ubiquitin ligase RING subunit of SCF $[1,8]$. Skp1 binds to the F-box protein, while RBX1, recruits the E2 ubiquitin-conjugating enzyme, which acts as a 'ubiquitin carrier' and is guided by the E3 ubiquitin ligase to the target substrate [1, 9]. (Fig. 1). The SCF complex is highly conserved among eukaryotes, which allows the transplant of specific Fbox proteins from one organism to another to form a functional SCF complex that can direct the degradation of proteins tagged with the target degron $[1,3,4]$. In the $C$. elegans AID system, the exogenous F-box protein used is the Arabidopsis thaliana transport inhibitor response 1 (TIR1) protein [2]. TIR1 recognizes tagged substrates and mark them for degradation only in the presence of the plant phytohormone, auxin (indole-3-acetic acid, or IAA) [1, 2, 3, 4]. Therefore, with an endogenous degron-tagged target protein and exogenous TIR1 expression, addition of auxin triggers rapid and efficient degradation of the targeted protein $[2,3,4]$.

The AID system is not only highly effective in degrading target proteins, but the system has proven to be specific, quick, and reversible in a multitude of 
species $[2,3,4,10,11,12]$. The first successful use of the AID system in nonplant cells was completed by Nishimura et al. using budding yeast and human cells. These cells, which contained an auxin degron on a GFP-NLS (SV40 nuclear localization signal), experienced near complete GFP signal loss within 30 minutes of exposure to an auxin treatment [10]. In these same cells it was observed that after removal from an auxin treatment GFP signal would begin to recover [10]. Since Nishimura's experiment, the system has successfully been used in fission yeast, Drosophila melanogaster, Caenorhabditis elegans, zebrafish, and mouse oocytes [2, 3, 4, 11, 12].

In C. elegans, the AID system successfully destroys degron-tagged nuclear and cytoplasmic proteins in both somatic and germline tissues across developmental stages [2]. Target proteins were depleted within two hours of auxin treatment, displaying not only the system's speed, but it's ability for continued use in C. elegans [2]. Developmental stage affects the rate of degradation, as degron-tagged protein degrade faster in young larvae as compared to adults [2]. Both degradation rate and recovery rate depended largely on the concentration of auxin used, with greater auxin concentrations resulting in faster degradation, but slower recovery [2]. In smaller concentrations, recovery was detectable by GFP fluorescence within two hours of removal from an auxin treatment [2]. After seven hours the fluorescence was observed to reach half the level seen in untreated animals [2]. No adverse side effects were observed following exposure to auxin or expression of TIR1 [2]. There was no 
effect on viability, brood size, developmental rate, or morphology [2]. These results established that the AID system as a capable and effective method for rapid protein degradation in C. elegans.

\subsubsection{DREAM Complex}

The 8-subunit DREAM (dimerization partner, retinoblastoma (Rb)-like, E2F, multivulval class B (MuvB)) complex is a transcriptional repressor complex (Fig. 2) that is highly conserved across vertebrates, Drosophila, and C. elegans [5]. In mammals, the DREAM complex represses cell cycle genes in G0, or cell cycle quiescence $[5,13,14]$. However, during late $\mathrm{G} 1$ and $\mathrm{S}$ phase of the cell cycle, MuvB dissociates from DREAM and forms the MMB transcriptional activator complex with the BMYB transcription factor $[5,13,14]$. DREAM maintains quiescence by repressing G1/S genes through binding E2F or E2F/CLE DNA promoter elements and repressing G2/M genes by binding CHR or CHR/CDE DNA promoter elements $[5,13,14]$. When the RB-like subunits, E2F, and DP are released, the MuvB complex, made up of LIN9, LIN37, LIN52, LIN54, and RBBP4 (LIN-53 in C. elegans), begins to coordinate cell cycle gene activation by associating with MYB in the late $S$ and early $G 2$ phase $[5,13,14]$. Further association with the transcriptional activator FOXM1 in late G2/M phase drives maximal expression of G2/M genes [5, 13, 14].

In contrast to mammalian DREAM, the homologous DRM complex in $C$. elegans and MuvB in particular, acts solely as a transcriptional repressor complex [6]. This is likely because C. elegans LIN-52 does not possess the 
phosphorylation switch of mammalian LIN52 and to date there has not been a BMYB homolog found in C. elegans [6]. In addition, this complex protects developing somatic cells by repressing germline genes and plays a role in cell fate specification through its antagonization of Ras signaling during the animal's vulval development $[15,16]$. The $C$. elegans DRM complex presents a unique opportunity to examine how DREAM represses target genes. As this complex, and MuvB, act solely in a repressive fashion, disruption of MuvB only eliminates repression in C. elegans [6]. However, in mammals, because MuvB plays a role in both the repressive DREAM complex and MMB activator complex, knockout of MuvB would not only eliminate gene repression, but activation as well, leading to negative effects on cell viability $[6,13,14,19]$.

\subsubsection{Dysfunction of MuvB and DREAM}

The DREAM complex and its MuvB subcomplex play a key role in the maintenance of cell cycle progression and cellular development. Across many species, loss of DREAM or MuvB can lead to cancer, abnormal bone growth, embryonic lethality, and the misexpression of genes $[16,17,18,19,20,21]$. At the least, these consequences negatively affect an organism's quality of life, and at their worst have proven fatal.

In cancer, DREAM complex-mediated gene repression is often disrupted, with MuvB constitutively adopting its activator function $[17,18]$. Constitutive MuvB activation is often caused by overexpression of the genes BMYB and FOXM1 $[17,18]$. In an examination of meningiomas, the most common primary 
tumors of the brain and central nervous system, only aggressive and malignant tumors lost DREAM complex function, while the benign and recurrent benign tumors still maintained normal DREAM function [17]. The malignant tumors overexpressed both FOXM1 and BMYB, which are hypothesized to be forcing MuvB into its gene activation functions. Constitutive MuvB-mediated gene activation then establishes continued cell proliferation [17]. Further study into BMYB found that its overabundance in cancers causes interference with LIN52 phosphorylation, which leads to an increased stability and abundance of LIN52, ultimately resulting in continued proliferation as a result of disrupted DREAM assembly [18]. Looking beyond cancer, mice deficient of p130, a retinoblastomalike protein, develop defects in endochondral bone formation and while they survive to parturition, all die within the first 1.5 days of life [19].

Loss of MuvB has been shown to have deleterious effects [20,21]. In mice, after detection in the blastocyte stage, LIN9 deficient embryos die shortly after implantation [20]. Prior to death, LIN-9 deficient embryos are considered highly abnormal with a distinct lack of development of the three germ layers, the amnion, chorion, and allantois [20]. In addition, the LIN9 loss causes a delayed entry into mitosis, a significant increase in binuclear cells, micronucleation, nuclei with multiple lobes, and doughnut-shaped nuclei [20]. LIN9 is also critical in adult mice, in LIN9 conditional knockouts, adults die within seven days of its depletion [20]. Conditional loss of LIN9 results in rapid atrophy of the mouse intestinal epithelium and a substantial decrease in cell proliferation [20]. Furthermore, LIN9 
appears to play a critical role in the regulation of embryonic stem cells [21]. Embryonic stem cells deficient in LIN9 experience impaired proliferation and embryoid body formation, with an increase in polyploid cells and an overabundance of cells in the G2 and M phases [21].

In C. elegans, disruption of DRM components leads to larval arrest and gene misexpression $[6,16,22]$. Loss of LIN-35, LIN-9, LIN-37, and LIN-54 causes a strong high temperature larval arrest phenotype, where at $26^{\circ} \mathrm{C}$ the worms do not develop past their first larval (L1) stage [16]. In these mutants, ectopic P-granule expression in the intestines was found to be correlated with their high temperature arrest phenotype [16]. In addition, at $26^{\circ} \mathrm{C}$, deficiency in LIN-54 and LIN-35 results in significant delays in chromatin compaction, which may allow for abnormal expression of germline genes in somatic cells [22]. In a LIN-35 null mutant, loss of the pocket protein impairs, but does not eliminate, chromatin association for the E2F-DP and MuvB subcomplexes [6]. Loss of LIN35 does cause the upregulation of many DREAM target genes; however, additional upregulation of DREAM target genes was observed when the LIN-35 null was coupled with depletion of MuvB [6]. In LIN-35 nulls with depletion of E2F-DP upregulation did not change, giving support to the belief that MuvB mediates the repression of DRM target genes [6]. As $C$. elegans MuvB has been shown to take on a purely repressive form, as opposed to dual-acting mammalian MuvB, it displays great promise as a model system for 
understanding how the DREAM complex mediates repression of DREAM target genes $[5,6]$.

\subsubsection{STUDY HYPOTHESIS}

In this project, we sought to assess how MuvB functions as a transcriptional repressor in C. elegans DRM. As outlined above, MuvB adopts a dual role in mammalian cells, repressing or activating target genes depending on the context of its protein associations [5]. The complexities of MuvB's role during the mammalian cell cycle, mean that loss-of-function analysis cannot ascertain how the complex functions as a repressor. In contrast, in C. elegans, MuvB appears to function solely as a repressor [6]. Therefore, targeted disruption of MuvB via degradation of two of its key subunits, LIN-9, the core protein of MuvB, and LIN-54, the sole DNA binding protein of MuvB, would cause significant upregulation of DRM target genes [14, 23]. This would further cement MuvB as

the key transcriptional repressor in $C$. elegans DRM and also suggest that individually LIN-9 and LIN-54 play a critical role in the repression of DREAM target genes.

To test our hypothesis, we utilized the AID protein degradation system that is not only effective, but highly specific, fast acting, and reversible [1, 2, 3, 4]. By overexpressing a TIR1 transgene in worm strains with GFP-degron-tagged LIN54 or LIN-9, we rapidly depleted the MuvB subunits after a simple addition of auxin. With the AID system, we performed one 6-hour and one 24 -hour auxin treatment time courses. We observed the consequence of MuvB subunit 
depletion on DRM target gene transcripts using quantitative PCR (qPCR). Using qPCR, we examined the relative quantity of three house-keeping genes, act-1, act-2, and tba-1, and three known DRM targets, air-1, cdk-1, and set-21. We expected that house-keeping genes would be unaffected following MuvB subunit degradation and DRM targets would be activated following MuvB subunit degradation. After auxin treatment, we observed that LIN-54 depletion caused DRM target activation but not LIN-9 depletion, suggesting that at least LIN-54 function is required for MuvB to mediate gene target repression. This study will further the understanding of the importance of individual proteins of the MuvB complex, and DRM as a whole, in transcriptional repression. 


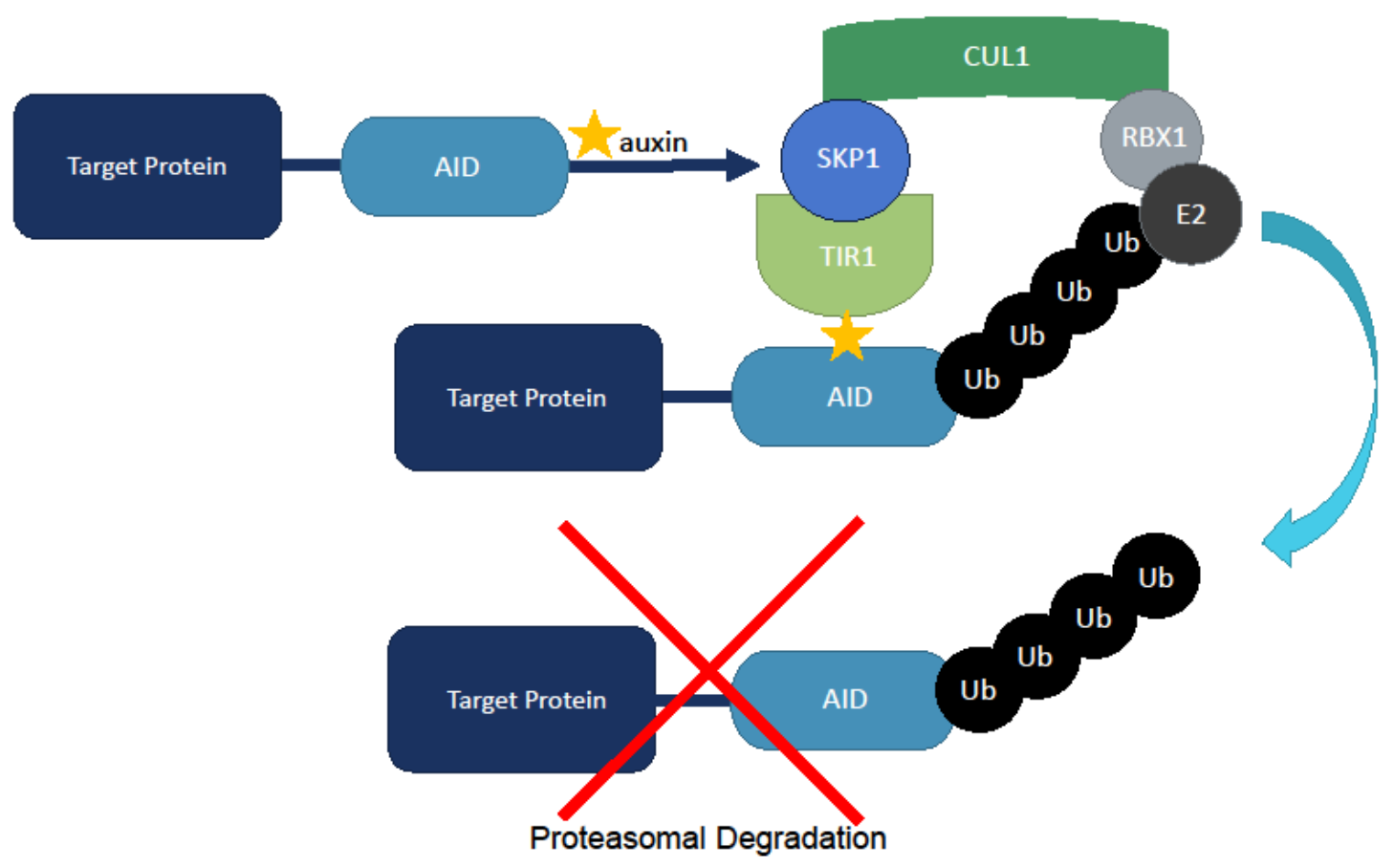

Figure 1 The auxin inducible degron (AID) system.

The AID system relies on the use of amino acide sequences called degrons fused to a target protein and the expression of the plant F-box protein TIR1. In the presence of auxin, TIR1, part of the SCF E3 ubiquitin ligase complex, will recognize the degron-tagged protein and mark it for degradation. 


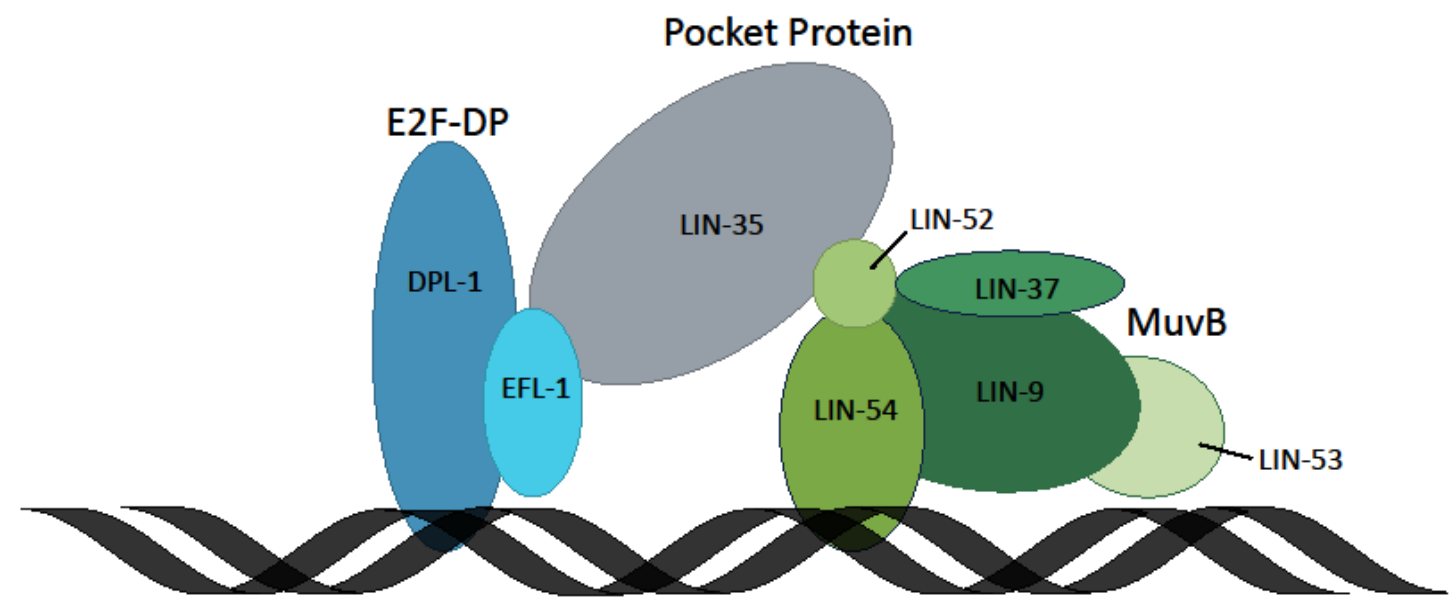

Figure 2 C. elegans DRM Complex

The DRM complex in C. elegans is a transcriptional repressor, consisting of eight proteins, split into three subunits, E2F-DP (DPL-1 and EFL-1), the pocket protein (LIN-35), and MuvB (LIN-9, LIN-37, LIN-52, LIN-53, LIN-54). Our project focuses on the effect on DRM target gene expression following degradation of LIN-54 and LIN-9 in C. elegans L1s over time. 


\subsection{MATERIALS AND METHODS}

\subsubsection{Worm Strains}

SS1369: lin-9(bn162(GFP::degron::3xflag::lin-9) III ; ieSi57(eft-

3p::TIR1::mRuby::unc-54 3' UTR + Cbr-unc-119(+) II

SS1371: lin-54(bn166(lin-54::GFP:::degron::3xflag) IV ; ieSi57(eft-

3p::TIR1::mRuby::unc-54 3' UTR + Cbr-unc-119(+) II

\subsubsection{Liquid Worm Growth}

Rapid expansion of worms was done using a liquid growth protocol. This was done using S-medium, which consisted of $500 \mathrm{ml}$ of $1 \times \mathrm{S}$-Basal, $5 \mathrm{ml}$ of $1 \mathrm{M}$ potassium citrate $\mathrm{pH} 6,5 \mathrm{ml}$ of $100 x$ trace metals, $1.5 \mathrm{ml}$ of $1 \mathrm{M} \mathrm{MgSO}_{4}, 1.5 \mathrm{ml}$ of $1 \mathrm{M} \mathrm{CaCl}_{2}$, and $500 \mu \mathrm{l}$ of $5 \mathrm{mg} / \mathrm{ml}$ cholesterol. To the S-medium, $10 \mathrm{ml}$ of HB101 was added to serve as the worms' food source. Six starved plates of $L 1$ worms were added to the solution by rinsing the plates with $1 \times$ S-Basal. These worms were then allowed to grow to gravid adults over a two- to three-day time period in an incubator set to $20^{\circ} \mathrm{C}$ shaking at $200 \mathrm{RPM}$.

\subsubsection{Obtaining L1s}

After growing to adulthood, worms were transferred into a $50 \mathrm{ml}$ tube from the liquid growth solution. To concentrate the worms to a single tube, the $200 \mathrm{~mL}$ culture was centrifuged $50 \mathrm{~mL}$ at a time at 1500 RPM for two minutes at $4^{\circ} \mathrm{C}$ to form a pellet and excess liquid was drawn off. Once concentrated, the pellet was washed $2-3$ times in deionized water, being centrifuged at 1500 RPM for two 
minutes at $4^{\circ} \mathrm{C}$ with the liquid drawn off for each wash. After the deionized water washes, the pellet was washed once with 1x S-Basal and centrifuged at 1500 RPM for two minutes at $4^{\circ} \mathrm{C}$ with the excess liquid removed. To obtain the embryos from the adult worms, a $50 \mathrm{ml}$ bleach solution of $0.7 \mathrm{~g} \mathrm{KOH}, 10 \mathrm{ml}$ bleach, and brought to $50 \mathrm{ml}$ with deionized water was created. $25 \mathrm{ml}$ of the bleach solution was added to every tube of adult worms. These tubes were then manually shaken for four minutes, with the degradation of the adult worms checked at four minutes. Degradation was monitored in two-minute intervals following the four minute check, once adults had fully disintegrated leaving only their embryos behind, the embryos were centrifuged at 1500 RPM for two minutes at $4^{\circ} \mathrm{C}$, with the bleach solution being removed. The embryos were then washed twice with deionized water and once with $1 \times$ S-Basal, following the same centrifuging protocol, with the liquid removed from the pellet after each wash. Following the washes, the pellet of embryos was resuspended in $50 \mathrm{ml}$ of $\mathrm{S}$ medium. The embryos were incubated overnight at $20^{\circ} \mathrm{C}$, shaking at $200 \mathrm{RPM}$. We obtained these embryos to synchronize the life stage of our experimental worms. We chose the first larval stage (L1) for our experiment as these worms can survive for an extended time without a food source and will not further their development until they receive adequate nutrition.

\subsubsection{Auxin Treatment Time Course}

The auxin treatment time courses to obtain RNA for $\mathrm{qPCR}$ were performed in triplicate for each timepoint. To start, $3.5 \mathrm{ml}$ of L1 worms in S- 
medium were treated with $35 \mu \mathrm{l}$ of $400 \mathrm{mM}$ Auxin, with the same concentrations being using for our vehicle control but substituting the $400 \mathrm{mM}$ Auxin treatment with an ethanol treatment. Two different time courses were performed, the first 24 hour timecourse with $0,1,3,6$, and 24 hour timepoints for the auxin treatment and controls, and the second 6 hour timecourse with $0,1,3$, and 6 hour timepoints for the auxin treatment and only one 6 hour ethanol vehicle treatment control. At each timepoint, $1 \mathrm{ml}$ of worms, which varied from $40,000 \mathrm{worms} / \mathrm{ml}$ to 50,000 worms $/ \mathrm{ml}$, were harvested and centrifuged for two minutes at 12,000 RPM. Once centrifuged the liquid was drawn off and $500 \mu \mathrm{l}$ of TRIzol was added. The samples were then stored at $-70^{\circ} \mathrm{C}$ until RNA isolation.

\subsubsection{RNA Isolation}

Once we were ready to perform the RNA extraction the tubes were thawed completely at room temperature. Once thawed, $100 \mu$ chloroform was added to the tubes, mixed, and incubated at room temperature for 10 minutes. After the incubation, the solution was centrifuged at $4^{\circ} \mathrm{C}$ for 15 minutes at 12,000 RPM. The top aqueous layer was transferred to an RNase free tube. To these tubes, a 1:1 ratio of isopropanol was added, mixed, and incubated for five minutes at room temperature. Following, samples were centrifuged for eight minutes at $4^{\circ} \mathrm{C}$ at $12,000 \mathrm{RPM}$. The liquid was removed, and $75 \%$ ethanol/nuclease-free water was added to wash. The tubes were then centrifuged for five minutes at 12,000 RPM. Following the spin, the liquid was removed, and the tubes were again centrifuged for one minute at 12,000 RPM. 
After, any remaining liquid was drawn off and the tubes were left open to air dry for no more than four minutes. After air drying for five minutes, nuclease-free water was added, and the samples were stored at $-70^{\circ} \mathrm{C}$ until reverse transcription was performed.

\subsubsection{Reverse Transcription}

Concentrations for RNA per sample were determined using a Nanodrop. For each sample, $500 \mathrm{ng}$ of RNA was reverse transcribed to complementary DNA (cDNA) using the High-Capacity cDNA Reverse Transcription Kit (ThermoFisher Scientific). All samples but the second run of LIN-54 underwent reverse transcription to cDNA. Following cDNA conversion or thawing on ice, tubes were then incubated at $65^{\circ} \mathrm{C}$ for 10 minutes and transferred to an ice bath. An RT-PCR master mix was created and to each tube $2 \mu \mathrm{l}$ of $10 \mathrm{x}$ RT buffer, 0.8 $\mu \mathrm{l}$ of $25 \mathrm{x}$ dNTP mix (100 mM), $2 \mu \mathrm{l}$ of random primers, $1 \mu \mathrm{l}$ of MultiScribe RTase and $3.2 \mu \mathrm{l}$ of nuclease-free water were added. The samples were mixed for short time using a tabletop centrifuge and run on an $A B I$ simpliAmp cycler. Once the run was complete the samples were moved into fresh tubes, diluted with $80 \mu \mathrm{l}$ ddH2O per sample, and refrigerated until quantitative PCR (qPCR) analysis.

\subsubsection{Quantitative PCR}

Dilutions for each primer set were made according to their reported concentrations with ddH2O prior to creating our qPCR plates. Our primer sets were act-1, act-2, tba-1, air-1, cdk-1, and set-21. Table 1 lists these primers and 
their corresponding DNA sequences. Using the dilutions, a master mix was created for each primer set. Each master mix contained $160 \mu$ of SYBR Green, $16 \mu \mathrm{l}$ of the forward primer, $16 \mu \mathrm{l}$ of the reverse primer and $96 \mu \mathrm{l}$ of $\mathrm{ddH}_{2} \mathrm{O}$. To each $2 \mu \mathrm{l}$ sample, $18 \mu \mathrm{l}$ of the master mix was added. Once the plate was completed it was centrifuged at 1500 RPM for two minutes and run using ABI QuantStudio 3. Relative gene quantities were obtained by finding $\Delta \mathrm{CT}$ of the genes, which we found by comparing all other tested genes to the house-keeping gene act-2, from the same timepoint and sample. Given $\Delta \mathrm{CT}$, we obtained relative quantity of the gene by finding $2^{-\Delta C T}$. The relative quantity from each sample per timepoint was averaged to give us the average relative quantity used in our figures. 


\begin{tabular}{|c|c|}
\hline Primer & Sequence \\
\hline act-1 $\mathrm{F}$ & ACGACGAGTCCGGCCCATCC \\
\hline act-1 $\mathrm{R}$ & GAAAGCTGGTGGTGACGATGGTT \\
\hline act-2 $\mathrm{F}$ & CGTCATCAAGGAGTCATGGTC \\
\hline act-2 $\mathrm{R}$ & TCAACACTGCCATCGCCGCC \\
\hline tba-1 $\mathrm{F}$ & TCCAAGCGAGACCAGGCTTCAG \\
\hline tba-1 $\mathrm{R}$ & ACGCCATACATTGTGCGGTA \\
\hline air-1 $\mathrm{F}$ & CCAGTTTGATTGGCGAACGG \\
\hline air-1 $\mathrm{R}$ & TTCAGAGTTCTCGGCACACC \\
\hline cdk-1 $\mathrm{F}$ & TTCGCGTTGAGACGAAGTGA \\
\hline cdk-1 $\mathrm{R}$ & AAATGTTGCGCGAACTGTCG \\
\hline set-21 $\mathrm{F}$ & GTCCGTGTACGTCTTTCCGT \\
\hline set-21 $\mathrm{R}$ &
\end{tabular}

Table 1 Primers and their corresponding DNA sequences 


\subsection{RESULTS}

\subsubsection{Auxin-induced degradation of LIN-54 does not affect expression of house-keeping genes}

As a control to compare to our DREAM target genes and to ensure that auxin treatment does not cause off target effects, we performed qPCR testing the transcript levels of the house-keeping genes act-1, act-2, and tba-1. Only data from the 6 hour time course was obtained for LIN-54. We chose act-2 as our reference gene to determine relative quantity of our experimental genes act-1 and tba-1. In our first run of act-1 and both runs of tba-1, we observed no significant difference in the relative quantity of our house-keeping genes (Fig. 3,

Fig. 4, Fig. 8). However, in our second run of act-1, some statistical significance was observed. In this run, act-1 was significantly higher in our 6-hour vehicle when compared to our 0-hour auxin control and 1-hour auxin timepoint. Our 3hour and 6-hour auxin treatment also showed significantly higher expression of act-1 in comparison to our 0 -hour and 1 -hour auxin treatment. The increase in act-1 over time in our second run suggest that these L1s may be experiencing a great deal of stress (Fig. 9).

\subsubsection{Auxin-induced degradation of LIN-54 results in activation of DREAM target genes}

We next tested transcript levels known DREAM target genes, air-1, cdk-1, and set-21, in degron-tagged LIN-54 L1 larvae in response to auxin treatment. 
We initially observed that air-1 (Fig. 10) and cdk-1 (Fig. 11) showed a steady, but insignificant, increase in the expression when compared to our 0-hour auxin and 6-hour vehicle controls. Therefore, we repeated the experiment and observed a statistically significance increase in expression of all 3 DREAM target genes

when compared to our 0-hour auxin and 6-hour vehicle controls (Fig. 10, Fig. 11, Fig. 12). In general our 6-hour vehicle experienced higher expression of all DREAM target genes than our 0-hour auxin control, at times even significantly higher. This suggests that the worms undergoing a 6-hour time course are under more stress than those that only experience a shorter time course. However, while expression in the 6-hour vehicle was higher than our 0-hour auxin control, it was across the board significantly less than our 6 -hour auxin treatment in our second run.

\subsubsection{Auxin-induced degradation of LIN-9 does not affect expression of house-keeping genes}

In LIN-9 we tested the transcript levels of the three house-keeping genes, act-1, act-2, and tba-1. We performed one 6-hour and one 24-hour auxin time course on degron-tagged LIN-9 L1 larvae, with gene transcript levels measured and treatment occurring at 0-hour, 1-hour, 3-hour, 6-hour, and 24-hour, for both auxin and the ethanol vehicle. We chose to only show results for the 24-hr ethanol vehicle. In both the 6-hour and 24-hour time courses, we chose act-2 as our standard comparison gene to calculate relative quantity of our experimental genes. As expected in both time course experiments, we observed that 
expression of tba-1 and act-1 was not consistently affected by auxin treatment (Fig. 13, Fig. 14, Fig. 18, Fig. 19). However, we observed that several time points showed significant changes in gene expression, when compared to 0-hour auxin or ethanol controls, but the change in expression levels was not reproducible between experiments. Therefore, we concluded that these significant changes were not a result of auxin treatment. In our 24-hour run of act-1 we noticed that our 24-hour ethanol vehicle had significantly higher expression of act-1 than our 0 -hour control. This suggests that treating L1s for such an extended time period may lead to great stress and it may be best to avoid long time courses in L1s.

\subsubsection{Auxin-induced degradation does not cause a significant effect on the expression of DREAM target genes}

Using act-2 transcript levels as a control, we measured the relative quantity transcript levels of the known DREAM target genes, air-1, cdk-1, and set-21. The degradation of LIN-9 following auxin treatment had no measurable effect on the expression of DREAM target genes. In contrast to our degrontagged LIN-54 results above, auxin treatment of degron-tagged LIN-9 did not cause an increase in DREAM target gene expression over time (Fig. 15, Fig. 16,

Fig. 17, Fig. 20, Fig. 21, Fig. 22). As shown by our figures for air-1, cdk-1, and set-21 for both our 6-hour and 24-hour time course, we did not observe any uniform increase in the expression of these genes over time. From this we conclude that LIN-9 may not play a large role in the repression of DREAM target genes. 


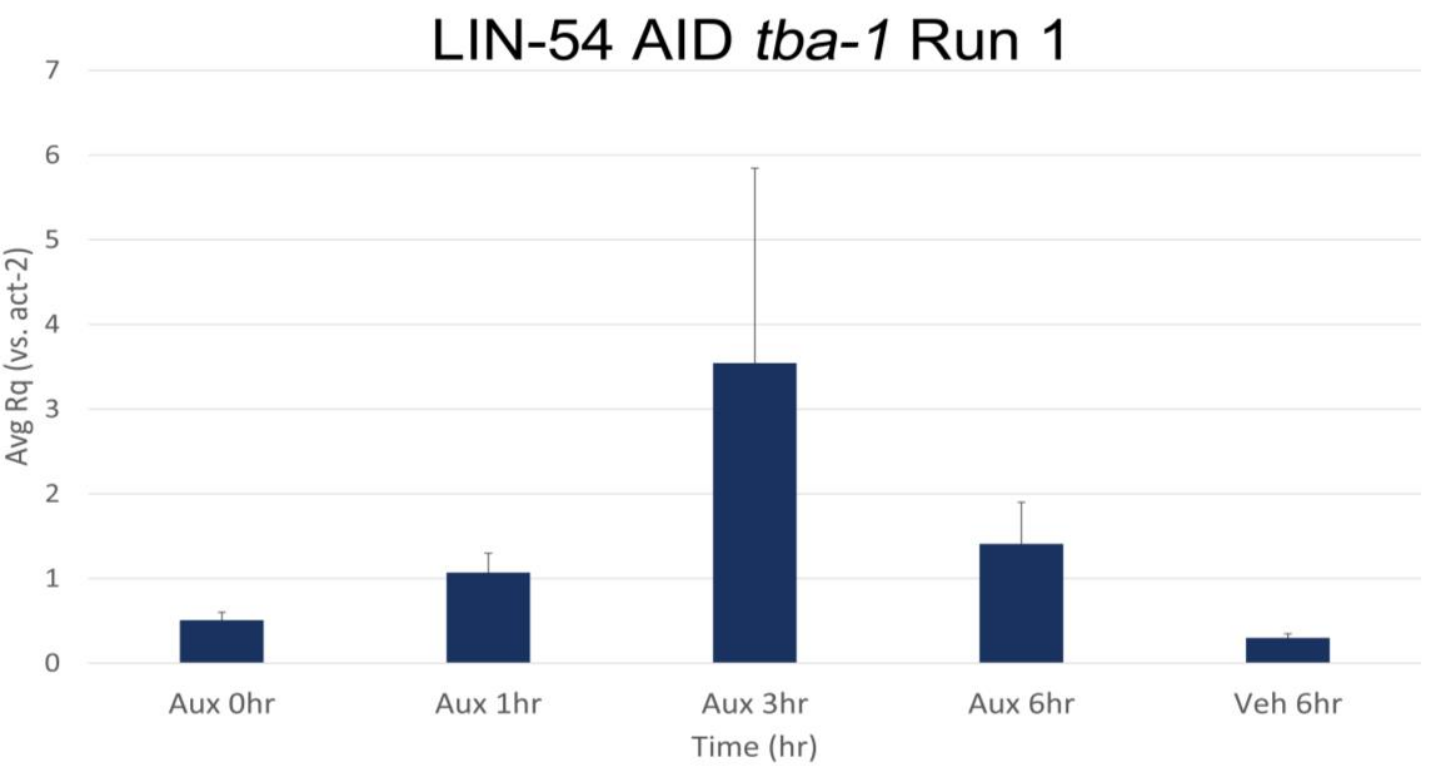

Figure 3 Average relative quantity (Rq) of tba-1 compared to act-2 over a 6hour auxin time course in LIN-54 degron-tagged L1 larvae.

Expression values from 3 biological replicates were averaged and are presented as the relative quantity $(\mathrm{Rq})$ compared to act-2. Synchronized L1 larvae were treated with $35 \mu \mathrm{l}$ of auxin for $0,1,3$, and 6 hours, with a 6 -hour ethanol treatment used as a vehicle control. Error bars indicate standard error of the mean. Significance was calculated using a student's T test $\left({ }^{*} p<0.05^{* *} p<0.01\right.$ experimental samples compared to 0 -hour auxin treatment, \# $p<0.05 \# \#<<0.01$ experimental samples compared to 6 -hour ethanol vehicle treatment). 


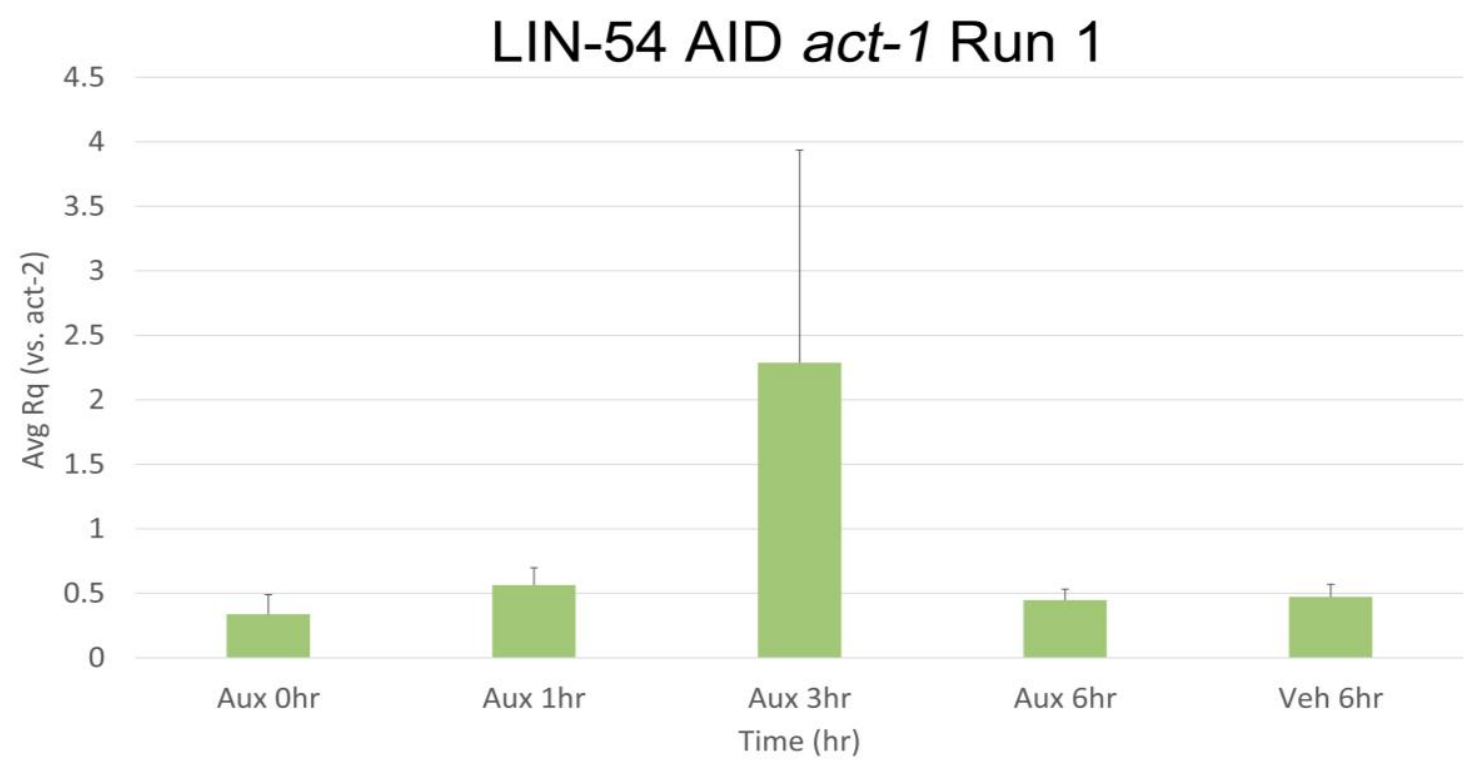

Figure 4 Average relative quantity (Rq) of act-1 compared to act-2 over a 6hour auxin time course in LIN-54 degron-tagged L1 larvae.

Expression values from 3 biological replicates were averaged and are presented as the relative quantity $(\mathrm{Rq})$ compared to act-2. Synchronized L1 larvae were treated with $35 \mu \mathrm{l}$ of auxin for $0,1,3$, and 6 hours, with a 6 -hour ethanol treatment used as a vehicle control. Error bars indicate standard error of the mean. Significance was calculated using a student's T test $\left({ }^{*} p<0.05{ }^{* *} p<0.01\right.$ experimental samples compared to 0 -hour auxin treatment, \# $p<0.05 \# \# p<0.01$ experimental samples compared to 6-hour ethanol vehicle treatment). 


\section{LIN-54 AID air-1 Run 1}

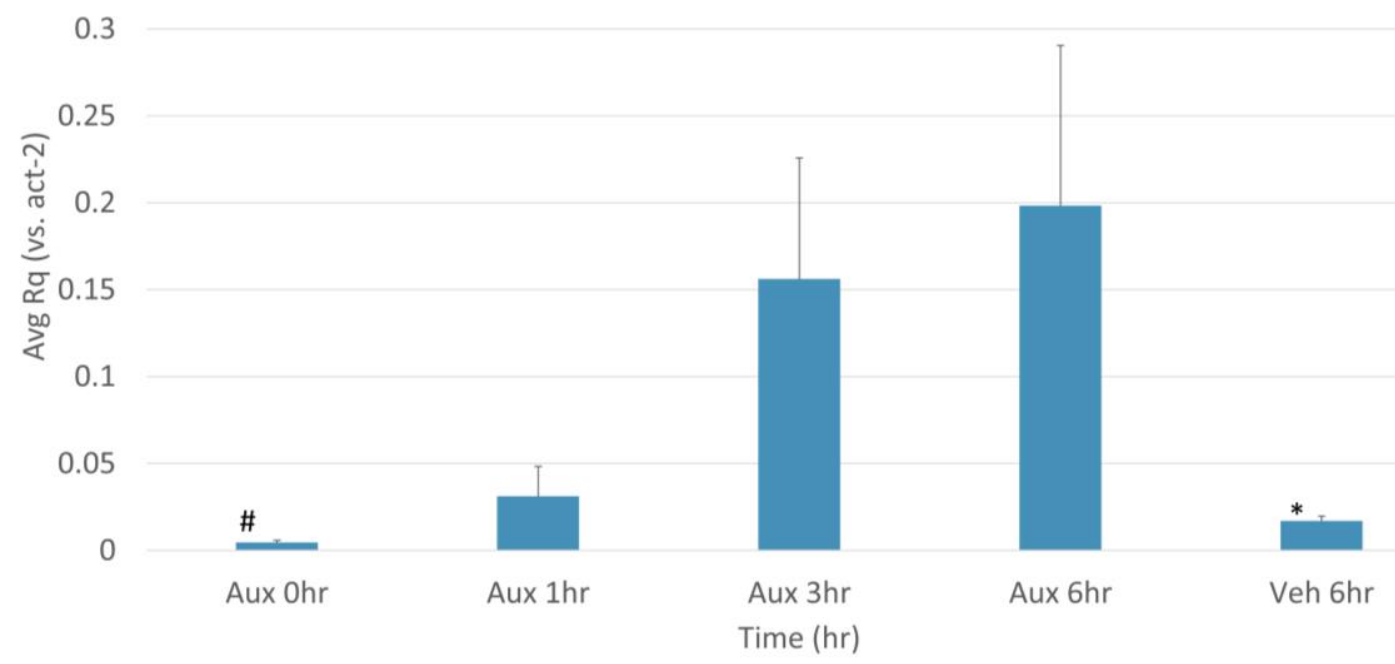

Figure 5 Average relative quantity $(\mathrm{Rq})$ of air-1 compared to act-2 over a 6hour auxin time course in LIN-54 degron-tagged L1 larvae.

Expression values from 3 biological replicates were averaged and are presented as the relative quantity $(\mathrm{Rq})$ compared to act-2. Synchronized L1 larvae were treated with $35 \mu \mathrm{l}$ of auxin for $0,1,3$, and 6 hours, with a 6 -hour ethanol treatment used as a vehicle control. Error bars indicate standard error of the mean. Significance was calculated using a student's T test $\left({ }^{*} p<0.05^{* *} p<0.01\right.$ experimental samples compared to 0 -hour auxin treatment, \# $p<0.05 \# \# p<0.01$ experimental samples compared to 6-hour ethanol vehicle treatment). 


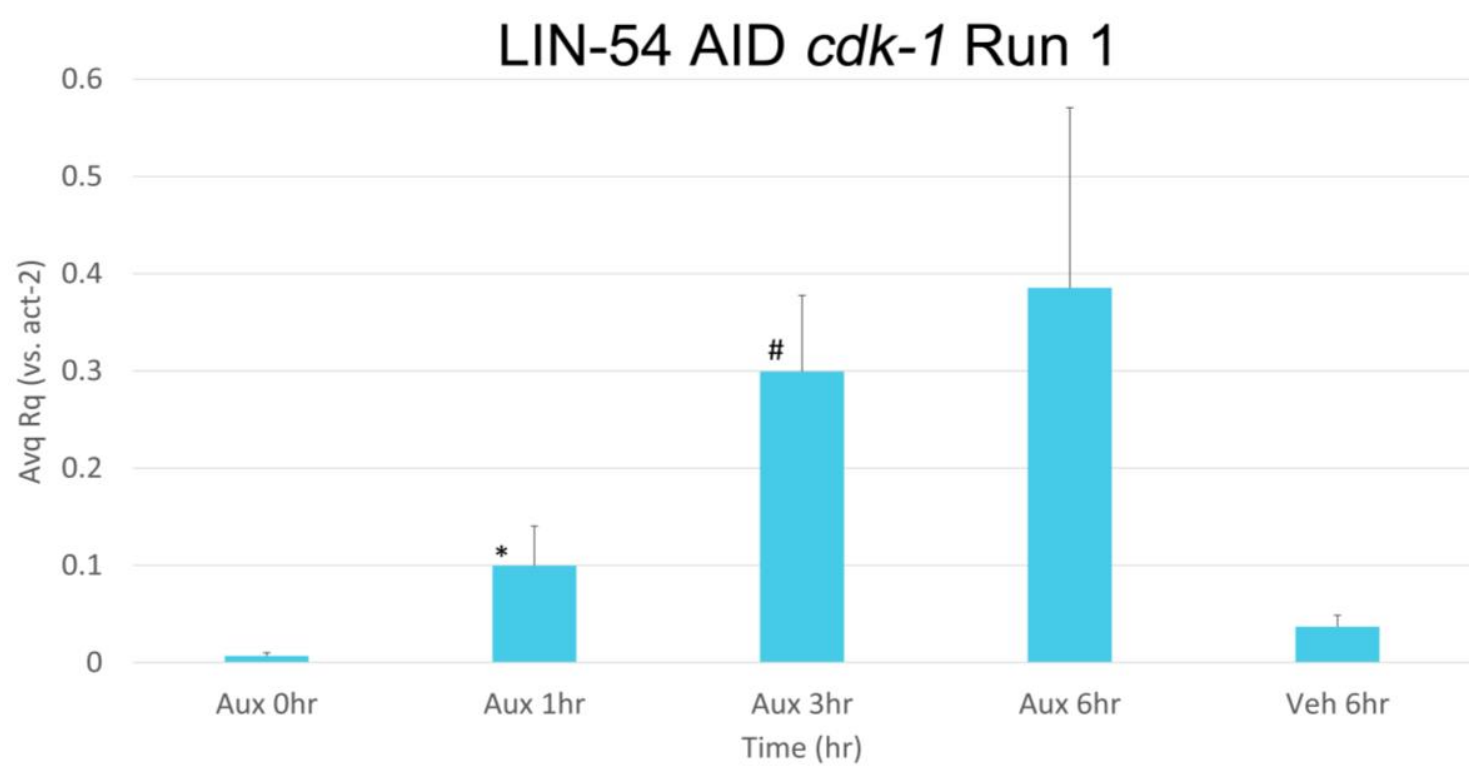

Figure 6 Average relative quantity (Rq) of $c d k-1$ compared to act-2 over a 6hour auxin time course in LIN-54 degron-tagged L1 larvae.

Expression values from 3 biological replicates were averaged and are presented as the relative quantity $(\mathrm{Rq})$ compared to act-2. Synchronized L1 larvae were treated with $35 \mu \mathrm{l}$ of auxin for $0,1,3$, and 6 hours, with a 6 -hour ethanol treatment used as a vehicle control. Error bars indicate standard error of the mean. Significance was calculated using a student's T test $\left({ }^{*} p<0.05{ }^{* *} p<0.01\right.$ experimental samples compared to 0 -hour auxin treatment, \# $p<0.05 \# \#<<0.01$ experimental samples compared to 6-hour ethanol vehicle treatment). 


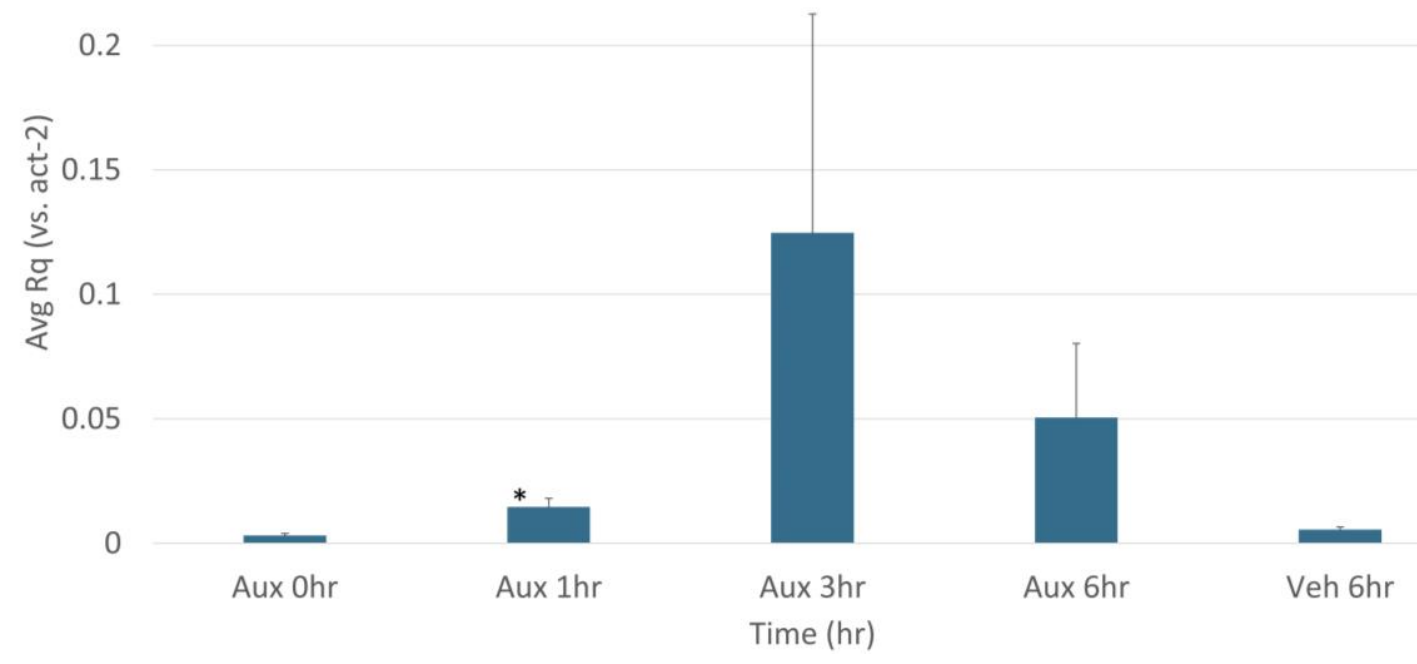

Figure 7 Average relative quantity $(\mathrm{Rq})$ of set-21 compared to act-2 over a 6hour auxin time course in LIN-54 degron-tagged L1 larvae.

Expression values from 3 biological replicates were averaged and are presented as the relative quantity $(\mathrm{Rq})$ compared to act-2. Synchronized L1 larvae were treated with $35 \mu \mathrm{l}$ of auxin for $0,1,3$, and 6 hours, with a 6 -hour ethanol treatment used as a vehicle control. Error bars indicate standard error of the mean. Significance was calculated using a student's T test $\left({ }^{*} p<0.05^{* *} p<0.01\right.$ experimental samples compared to 0 -hour auxin treatment, \# $p<0.05 \# p<0.01$ experimental samples compared to 6 -hour ethanol vehicle treatment). 
3

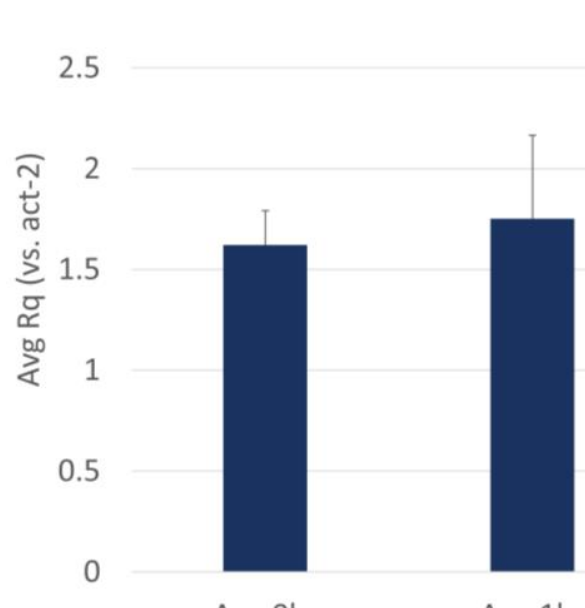

Aux Ohr

LIN-54 AID tba-1 Run 2

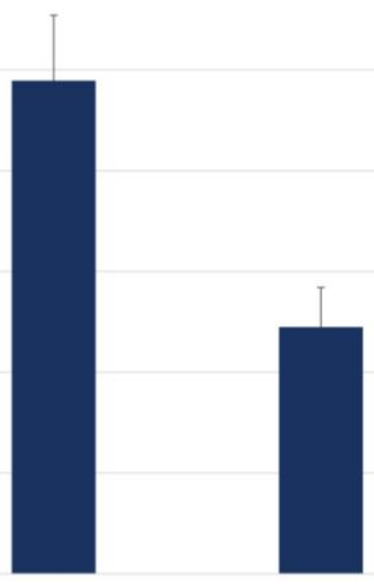

Aux 6hr

Figure 8 Average relative quantity $(\mathrm{Rq})$ of $t b a-1$ compared to act-2 over a 6hour auxin time course in LIN-54 degron-tagged L1 larvae.

Expression values from 3 biological replicates were averaged and are presented as the relative quantity $(\mathrm{Rq})$ compared to act-2. Synchronized L1 larvae were treated with $35 \mu \mathrm{l}$ of auxin for $0,1,3$, and 6 hours, with a 6 -hour ethanol treatment used as a vehicle control. Error bars indicate standard error of the mean. Significance was calculated using a student's T test $\left({ }^{*} p<0.05^{* *} p<0.01\right.$ experimental samples compared to 0 -hour auxin treatment, \# $p<0.05 \# \# p<0.01$ experimental samples compared to 6-hour ethanol vehicle treatment). 


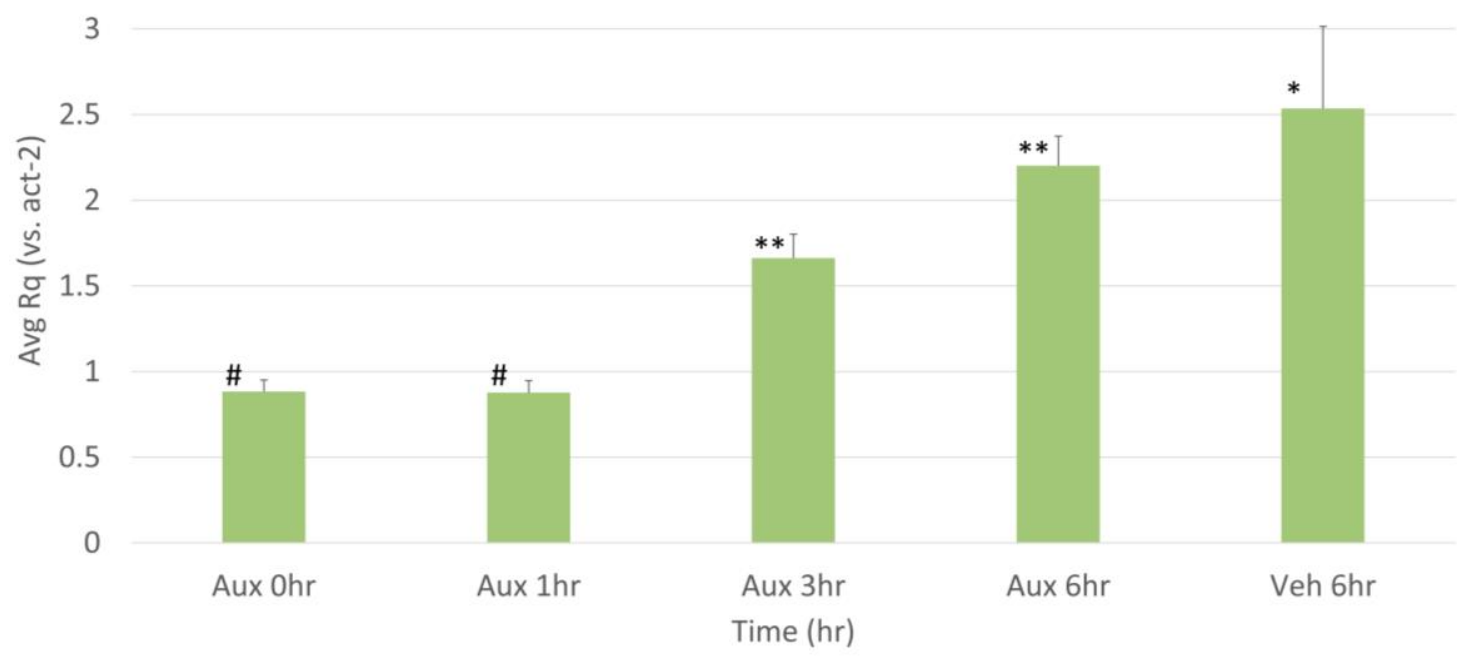

Figure 9 Average relative quantity $(\mathrm{Rq})$ of act-1 compared to act-2 over a 6hour auxin time course in LIN-54 degron-tagged L1 larvae.

Expression values from 3 biological replicates were averaged and are presented as the relative quantity $(\mathrm{Rq})$ compared to act-2. Synchronized L1 larvae were treated with $35 \mu \mathrm{l}$ of auxin for $0,1,3$, and 6 hours, with a 6 -hour ethanol treatment used as a vehicle control. Error bars indicate standard error of the mean. Significance was calculated using a student's T test $\left({ }^{*} p<0.05{ }^{* *} p<0.01\right.$ experimental samples compared to 0 -hour auxin treatment, \# $p<0.05 \# \# p<0.01$ experimental samples compared to 6-hour ethanol vehicle treatment). 
0.4

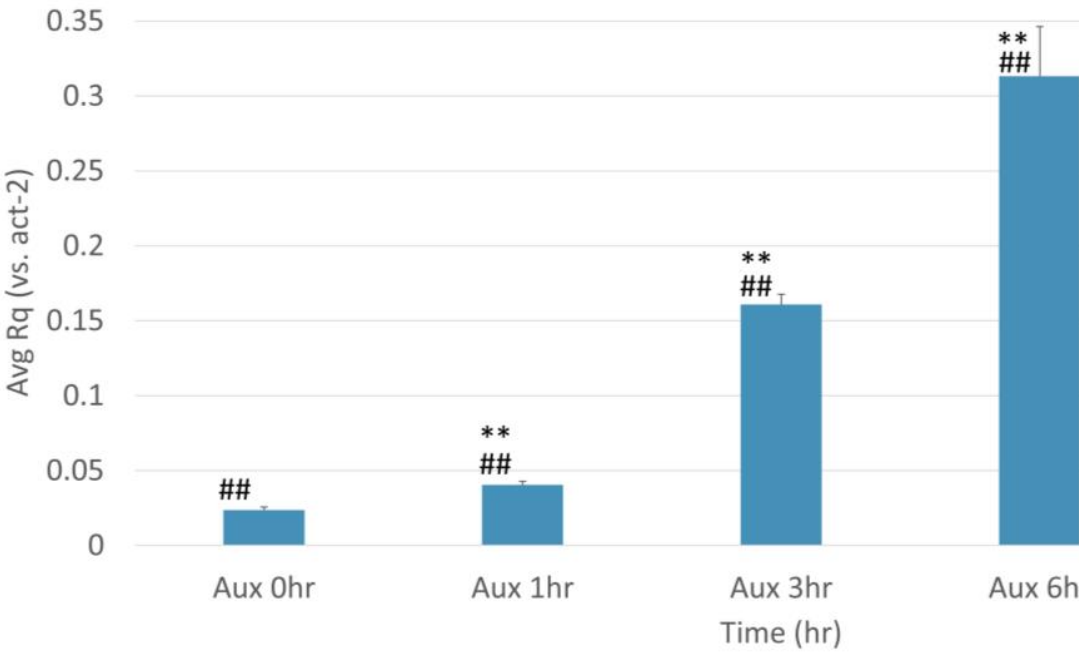

LIN-54 AID air-1 Run 2

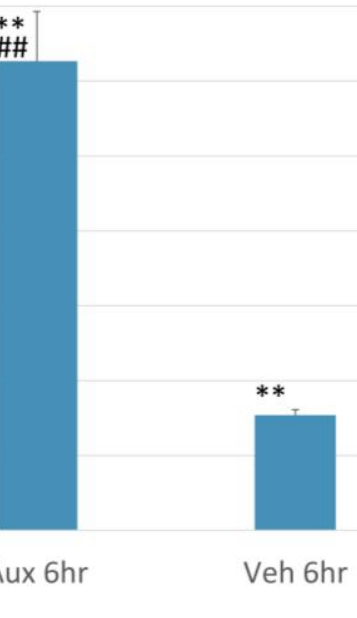

Figure 10 Average relative quantity $(\mathrm{Rq})$ of air-1 compared to act-2 over a 6hour auxin time course in LIN-54 degron-tagged L1 larvae.

Expression values from 3 biological replicates were averaged and are presented as the relative quantity $(\mathrm{Rq})$ compared to act-2. Synchronized L1 larvae were treated with $35 \mu$ of auxin for $0,1,3$, and 6 hours, with a 6 -hour ethanol treatment used as a vehicle control. Error bars indicate standard error of the mean. Significance was calculated using a student's T test $\left({ }^{*} p<0.05{ }^{* *} p<0.01\right.$ experimental samples compared to 0 -hour auxin treatment, \# $p<0.05 \# \# p<0.01$ experimental samples compared to 6-hour ethanol vehicle treatment). 


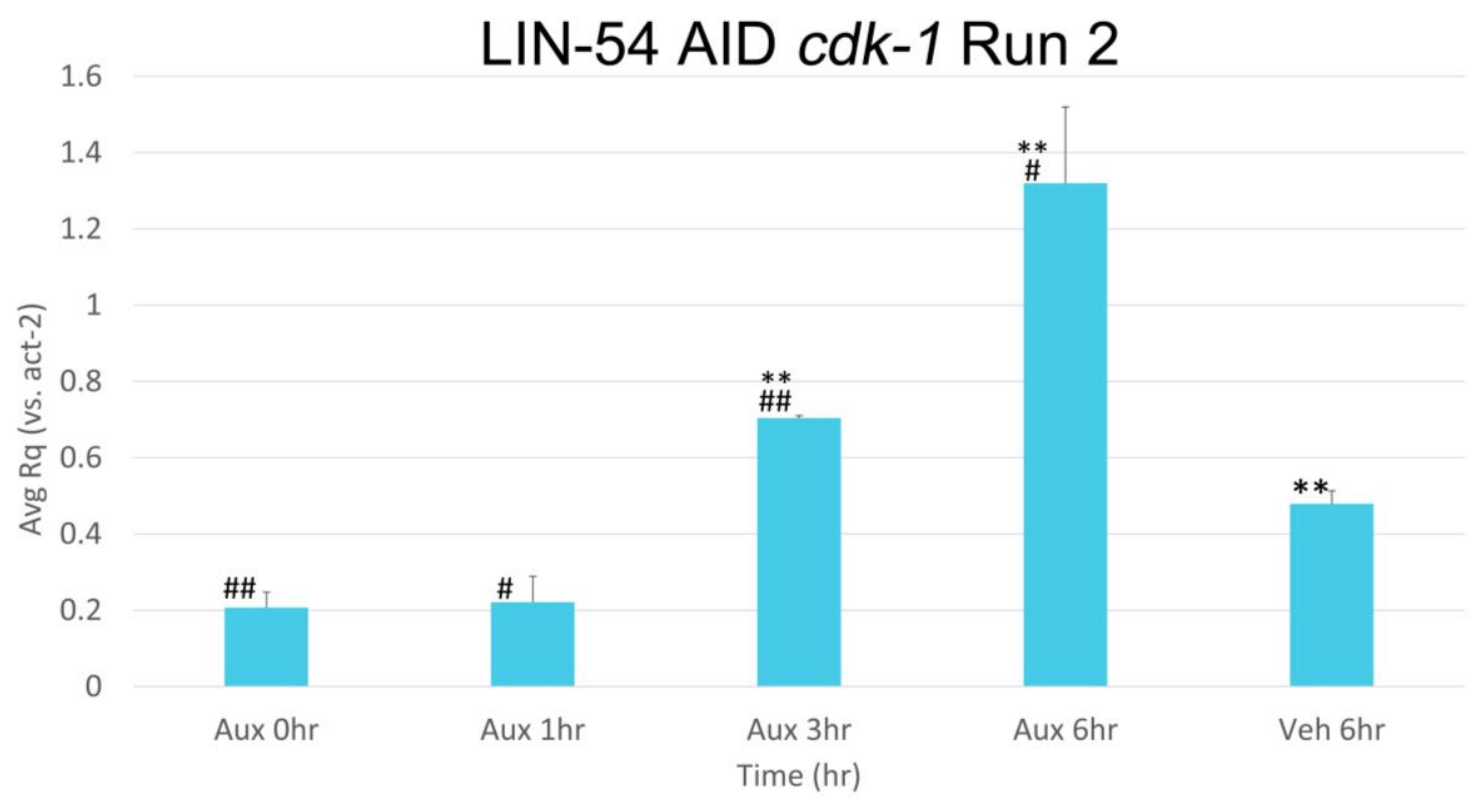

Figure 11 Average relative quantity (Rq) of $c d k-1$ compared to act-2 over a 6hour auxin time course in LIN-54 degron-tagged L1 larvae.

Expression values from 3 biological replicates were averaged and are presented as the relative quantity $(\mathrm{Rq})$ compared to act-2. Synchronized L1 larvae were treated with $35 \mu \mathrm{l}$ of auxin for $0,1,3$, and 6 hours, with a 6 -hour ethanol treatment used as a vehicle control. Error bars indicate standard error of the mean. Significance was calculated using a student's T test $\left({ }^{*} p<0.05{ }^{* *} p<0.01\right.$ experimental samples compared to 0 -hour auxin treatment, \# $p<0.05 \# \# p<0.01$ experimental samples compared to 6-hour ethanol vehicle treatment). 
LIN-54 AID set-21 Run 2

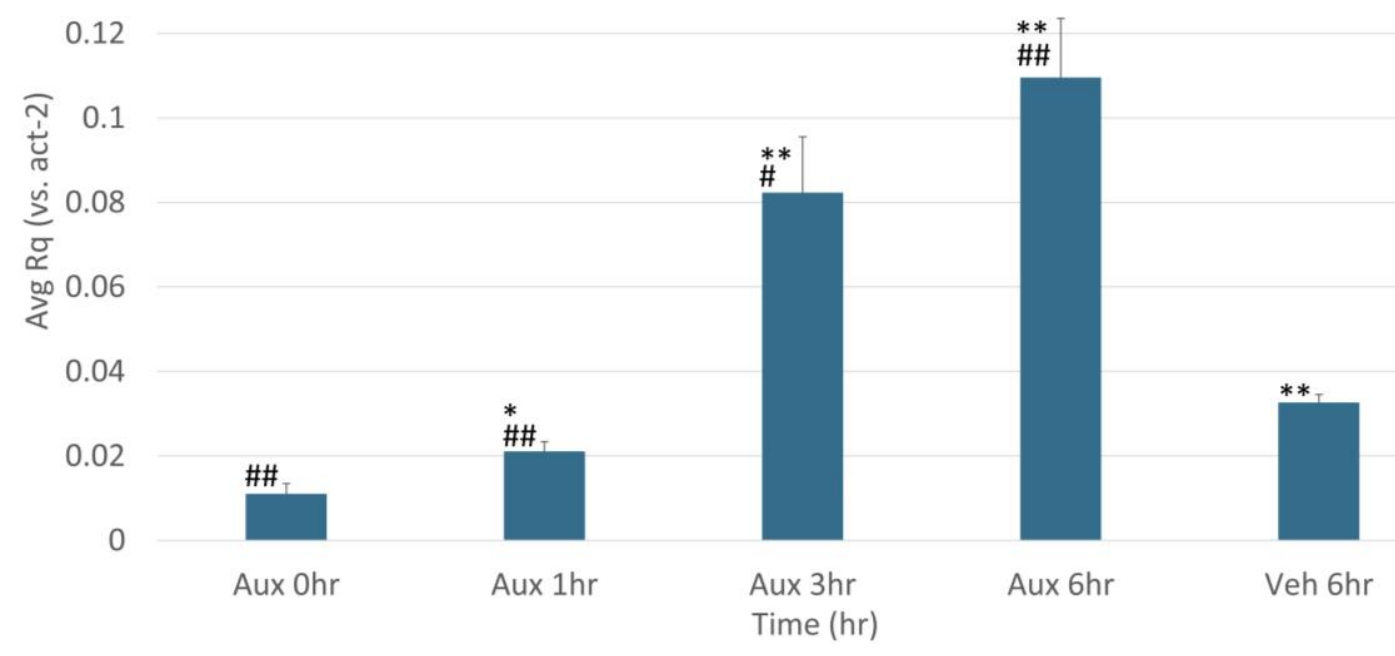

Figure 12 Average relative quantity $(\mathrm{Rq})$ of set-21 compared to act-2 over a 6hour auxin time course in LIN-54 degron-tagged L1 larvae.

Expression values from 3 biological replicates were averaged and are presented as the relative quantity $(\mathrm{Rq})$ compared to act-2. Synchronized L1 larvae were treated with $35 \mu \mathrm{l}$ of auxin for $0,1,3$, and 6 hours, with a 6 -hour ethanol treatment used as a vehicle control. Error bars indicate standard error of the mean. Significance was calculated using a student's T test $\left({ }^{*} p<0.05{ }^{* *} p<0.01\right.$ experimental samples compared to 0 -hour auxin treatment, \# $p<0.05 \# \#<0.01$ experimental samples compared to 6-hour ethanol vehicle treatment). 


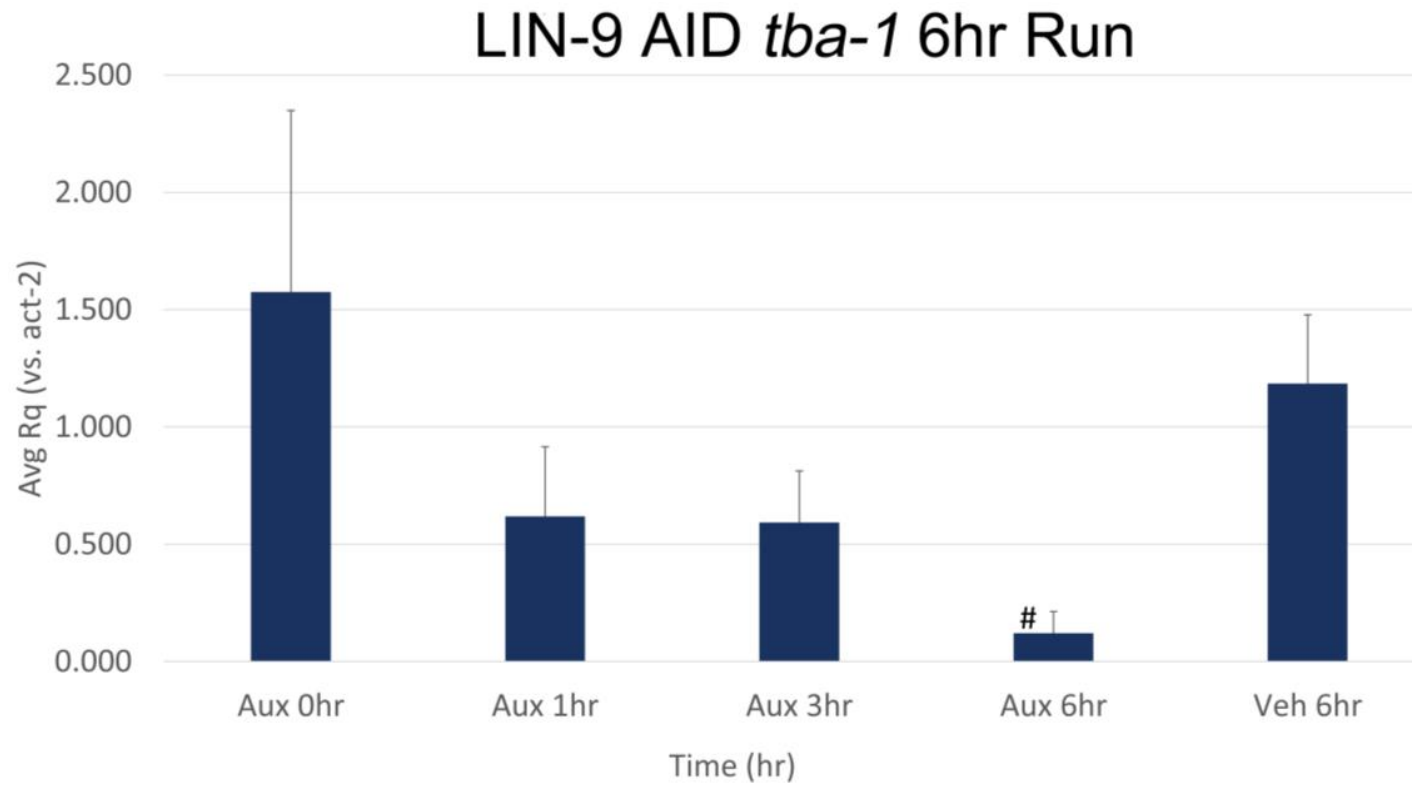

Figure 13 Average relative quantity $(\mathrm{Rq})$ of $t b a-1$ compared to act-2 over a 6hour auxin time course in LIN-9 degron-tagged L1 larvae.

Expression values from 3 biological replicates were averaged and are presented as the relative quantity $(\mathrm{Rq})$ compared to act-2. Synchronized L1 larvae were treated with $35 \mu \mathrm{l}$ of auxin for $0,1,3$, and 6 hours, with a 6 -hour ethanol treatment used as a vehicle control. Error bars indicate standard error of the mean. Significance was calculated using a student's T test $\left({ }^{*} p<0.05{ }^{* *} p<0.01\right.$ experimental samples compared to 0 -hour auxin treatment, \# $p<0.05 \# \#<<0.01$ experimental samples compared to 6-hour ethanol vehicle treatment). 


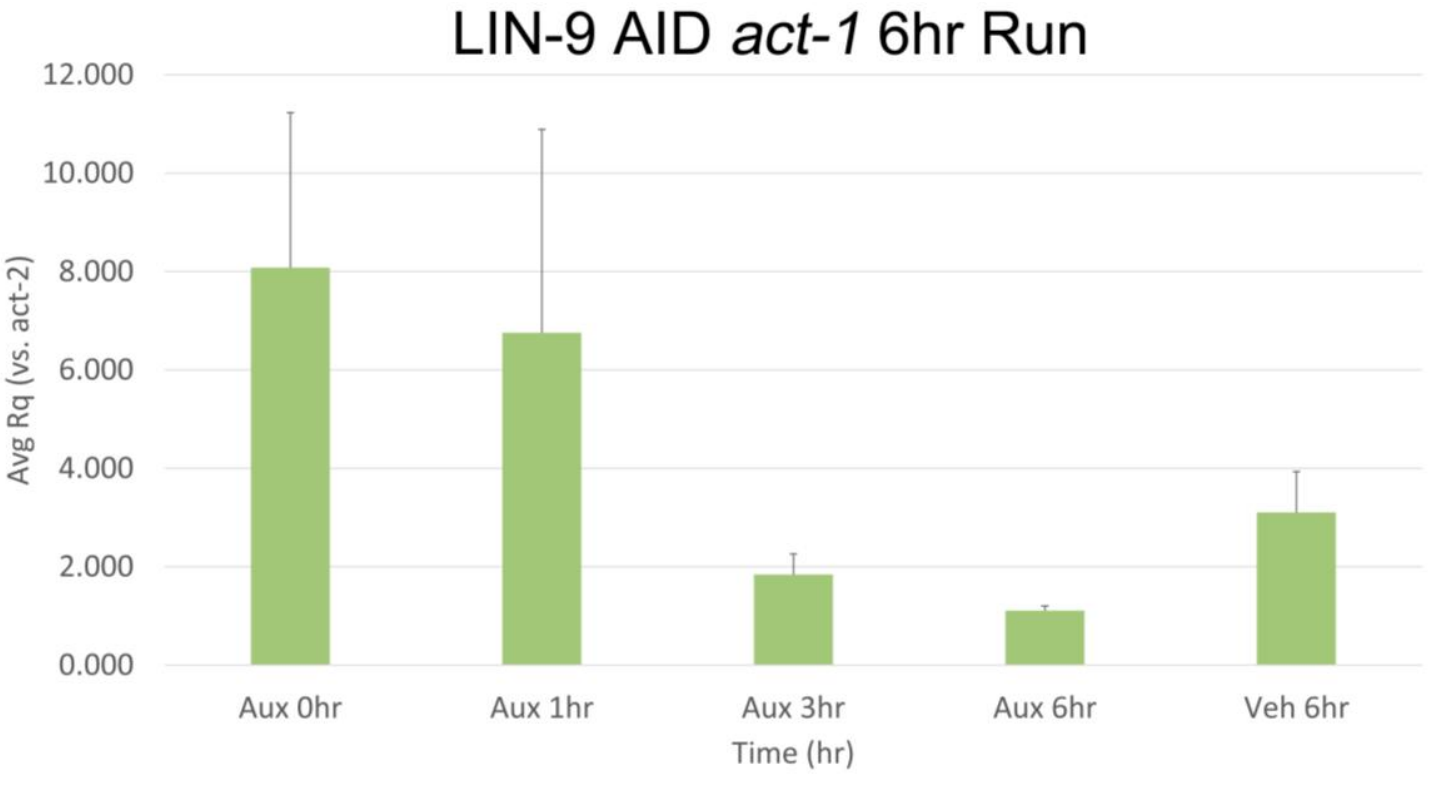

Figure 14 Average relative quantity $(\mathrm{Rq})$ of act-1 compared to act-2 over a 6hour auxin time course in LIN-9 degron-tagged L1 larvae.

Expression values from 3 biological replicates were averaged and are presented as the relative quantity $(\mathrm{Rq})$ compared to act-2. Synchronized L1 larvae were treated with $35 \mu \mathrm{l}$ of auxin for $0,1,3$, and 6 hours, with a 6 -hour ethanol treatment used as a vehicle control. Error bars indicate standard error of the mean. Significance was calculated using a student's T test $\left({ }^{*} p<0.05{ }^{* *} p<0.01\right.$ experimental samples compared to 0 -hour auxin treatment, \# $p<0.05 \#$ \# $<0.01$ experimental samples compared to 6 -hour ethanol vehicle treatment). 


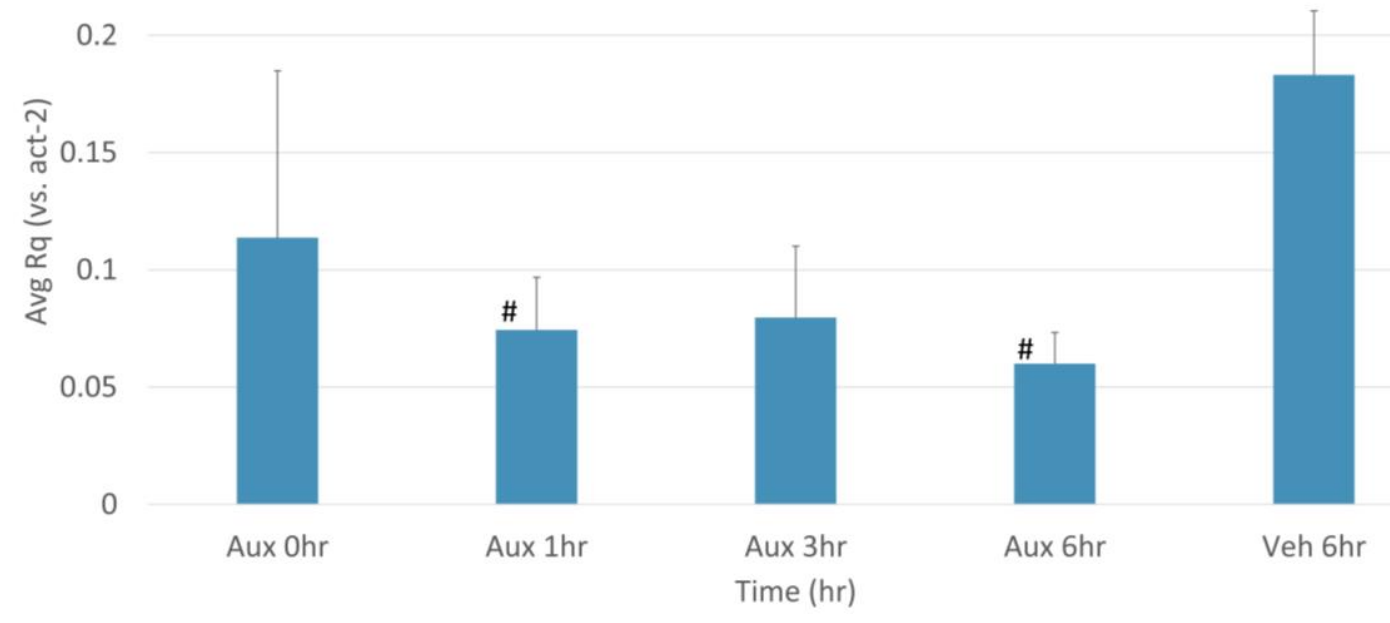

Figure 15 Average relative quantity $(\mathrm{Rq})$ of air-1 compared to act-2 over a 6hour auxin time course in LIN-9 degron-tagged L1 larvae.

Expression values from 3 biological replicates were averaged and are presented as the relative quantity $(\mathrm{Rq})$ compared to act-2. Synchronized L1 larvae were treated with $35 \mu$ of auxin for $0,1,3$, and 6 hours, with a 6 -hour ethanol treatment used as a vehicle control. Error bars indicate standard error of the mean. Significance was calculated using a student's T test $\left({ }^{*} p<0.05{ }^{* *} p<0.01\right.$ experimental samples compared to 0 -hour auxin treatment, \# $p<0.05 \#$ \# $<0.01$ experimental samples compared to 6-hour ethanol vehicle treatment). 


\section{LIN-9 AID cdk-1 6hr Run}

$$
\begin{aligned}
& 0.350 \\
& 0.300 \\
& \text { 突 } 0.250 \\
& \text { 它 } 0.200 \\
& \text { 通 } \\
& 0.100 \\
& 0.050 \\
& 0.000
\end{aligned}
$$

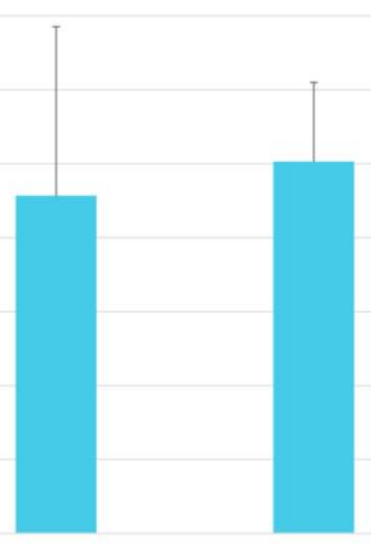

Aux $0 \mathrm{hr}$

Aux $1 \mathrm{hr}$

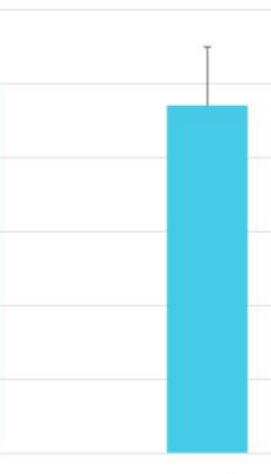

Aux $3 \mathrm{hr}$ Time (hr)

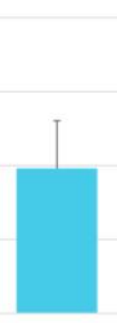

Aux $6 \mathrm{hr}$

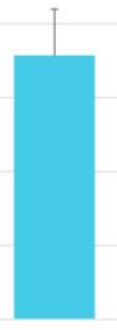

Veh 6hr

Figure 16 Average relative quantity (Rq) of $c d k-1$ compared to act-2 over a 6hour auxin time course in LIN-9 degron-tagged L1 larvae.

Expression values from 3 biological replicates were averaged and are presented as the relative quantity $(\mathrm{Rq})$ compared to act-2. Synchronized L1 larvae were treated with $35 \mu \mathrm{l}$ of auxin for $0,1,3$, and 6 hours, with a 6 -hour ethanol treatment used as a vehicle control. Error bars indicate standard error of the mean. Significance was calculated using a student's T test $\left({ }^{*} p<0.05{ }^{* *} p<0.01\right.$ experimental samples compared to 0 -hour auxin treatment, \# $p<0.05 \# \# p<0.01$ experimental samples compared to 6 -hour ethanol vehicle treatment). 


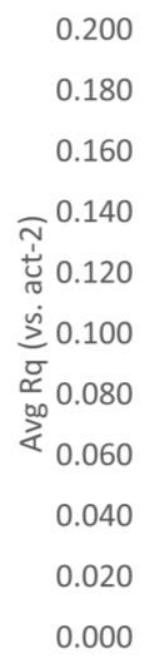

\section{LIN-9 AID set-21 6hr Run}

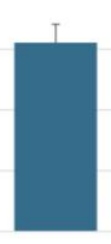

Aux Ohr

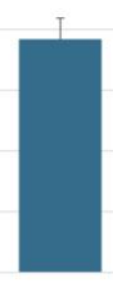

Aux $1 \mathrm{hr}$

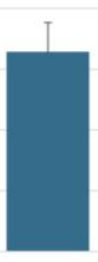

Aux $3 \mathrm{hr}$ Time (hr)

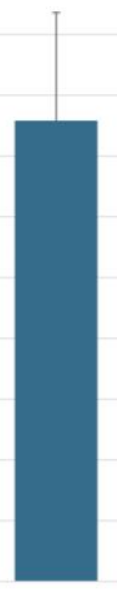

Veh $6 \mathrm{hr}$

Figure 17 Average relative quantity $(\mathrm{Rq})$ of set-21 compared to act-2 over a 6hour auxin time course in LIN-9 degron-tagged L1 larvae.

Expression values from 3 biological replicates were averaged and are presented as the relative quantity $(\mathrm{Rq})$ compared to act-2. Synchronized L1 larvae were treated with $35 \mu \mathrm{l}$ of auxin for $0,1,3$, and 6 hours, with a 6 -hour ethanol treatment used as a vehicle control. Error bars indicate standard error of the mean. Significance was calculated using a student's T test $\left({ }^{*} p<0.05{ }^{* *} p<0.01\right.$ experimental samples compared to 0 -hour auxin treatment, \# $p<0.05 \# \#<<0.01$ experimental samples compared to 6-hour ethanol vehicle treatment). 


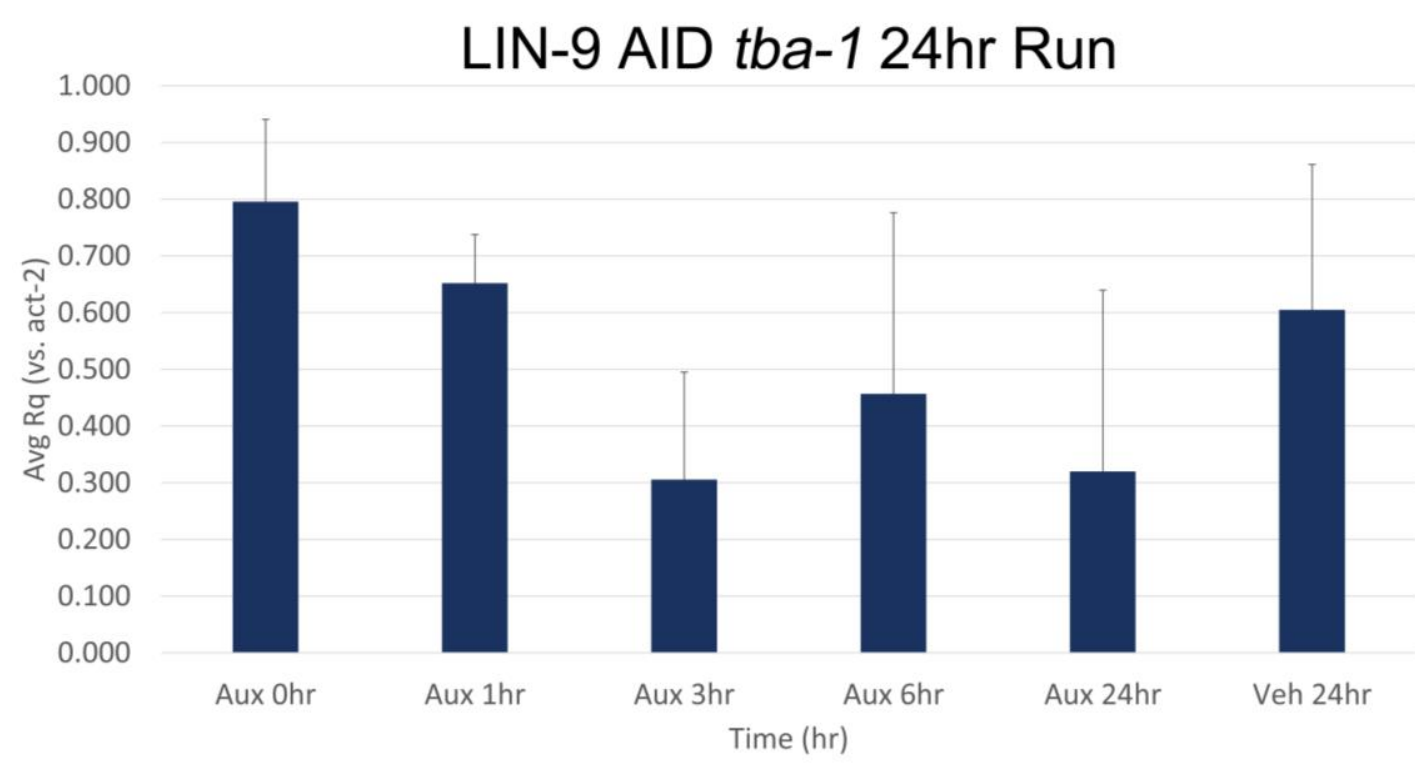

Figure 18 Average relative quantity (Rq) of tba-1 compared to act-2 over a 24-hour auxin time course in LIN-9 degron-tagged L1 larvae.

Expression values from 3 biological replicates were averaged and are presented as the relative quantity $(\mathrm{Rq})$ compared to act-2. Synchronized L1 larvae were treated with $35 \mu \mathrm{l}$ of auxin for $0,1,3$, and 6 hours, with a 6 -hour ethanol treatment used as a vehicle control. Error bars indicate standard error of the mean. Significance was calculated using a student's T test $\left({ }^{*} p<0.05{ }^{* *} p<0.01\right.$ experimental samples compared to 0 -hour auxin treatment, $\# p<0.05 \# \# p<0.01$ experimental samples compared to 6-hour ethanol vehicle treatment). 


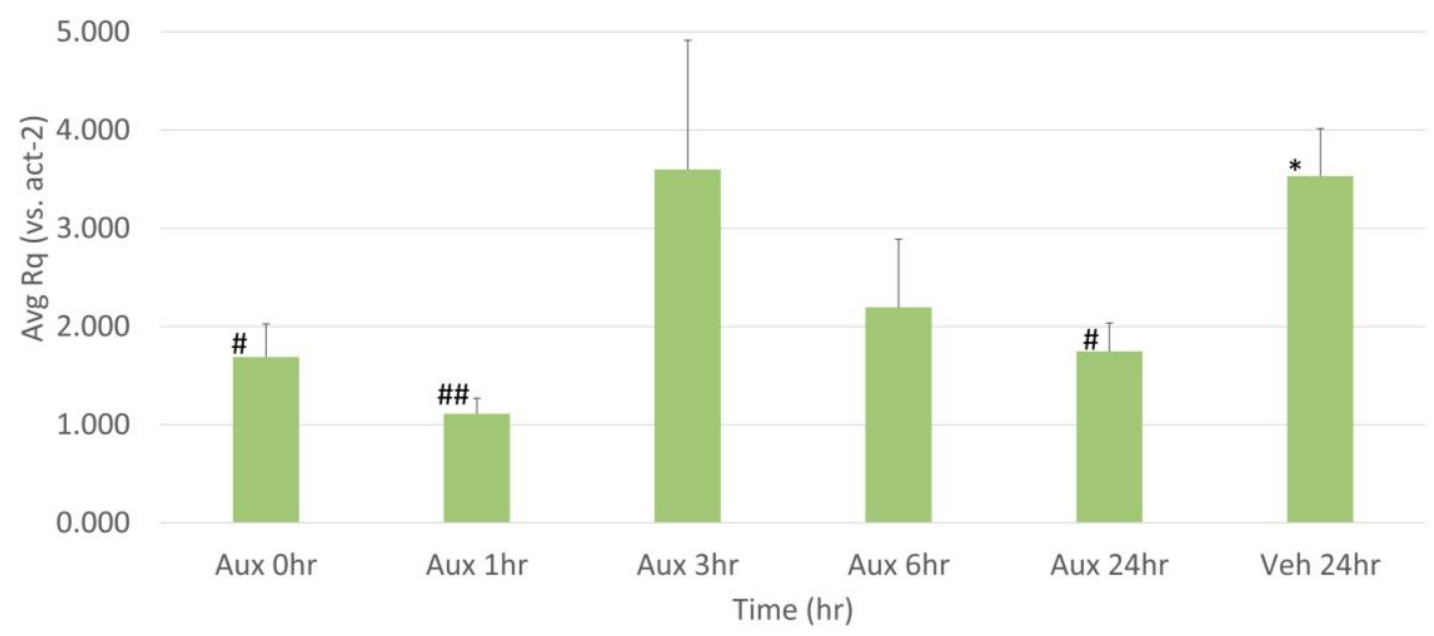

Figure 19 Average relative quantity $(\mathrm{Rq})$ of act-1 compared to act-2 over a 24hour auxin time course in LIN-9 degron-tagged L1 larvae.

Expression values from 3 biological replicates were averaged and are presented as the relative quantity $(\mathrm{Rq})$ compared to act-2. Synchronized L1 larvae were treated with $35 \mu \mathrm{l}$ of auxin for $0,1,3$, and 6 hours, with a 6 -hour ethanol treatment used as a vehicle control. Error bars indicate standard error of the mean. Significance was calculated using a student's T test $\left({ }^{*} p<0.05{ }^{* *} p<0.01\right.$ experimental samples compared to 0 -hour auxin treatment, \# $p<0.05 \# \#<0.01$ experimental samples compared to 6-hour ethanol vehicle treatment). 


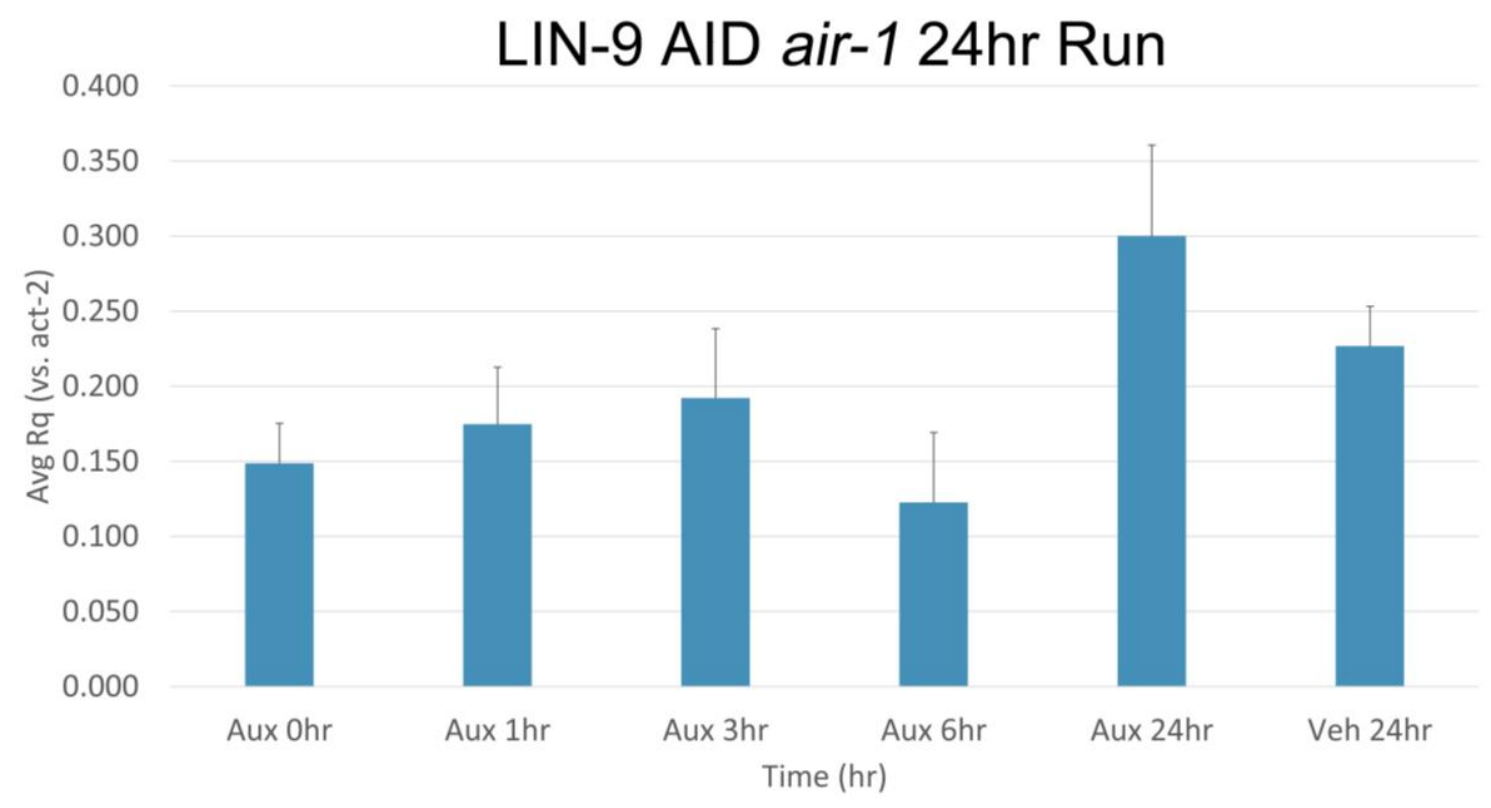

Figure 20 Average relative quantity $(\mathrm{Rq})$ of air-1 compared to act-2 over a 24hour auxin time course in LIN-9 degron-tagged L1 larvae.

Expression values from 3 biological replicates were averaged and are presented as the relative quantity $(\mathrm{Rq})$ compared to act-2. Synchronized L1 larvae were treated with $35 \mu \mathrm{l}$ of auxin for $0,1,3$, and 6 hours, with a 6 -hour ethanol treatment used as a vehicle control. Error bars indicate standard error of the mean. Significance was calculated using a student's T test $\left({ }^{*} p<0.05^{* *} p<0.01\right.$ experimental samples compared to 0 -hour auxin treatment, \# $p<0.05 \# \# p<0.01$ experimental samples compared to 6-hour ethanol vehicle treatment). 


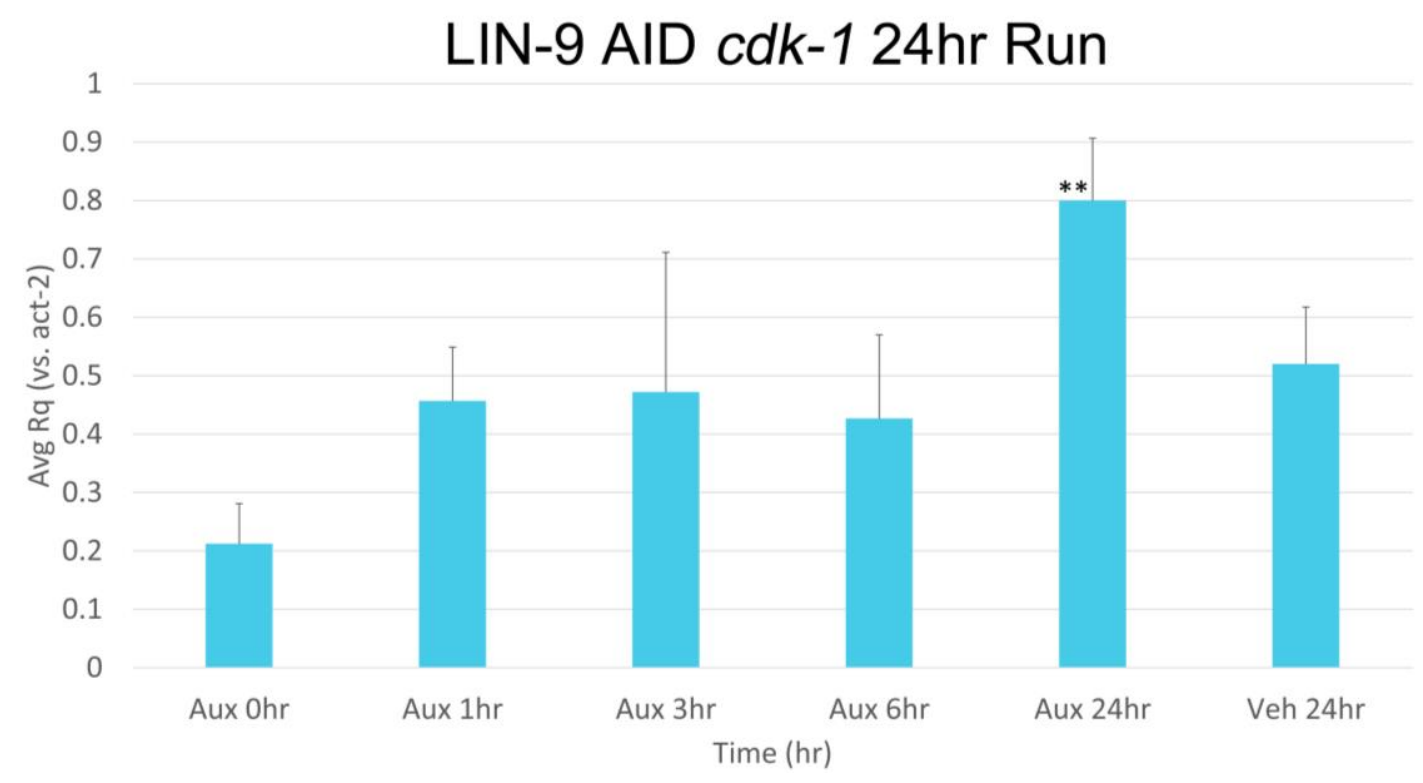

Figure 21 Average relative quantity $(\mathrm{Rq})$ of $c d k-1$ compared to act-2 over a 24-hour auxin time course in LIN-9 degron-tagged L1 larvae.

Expression values from 3 biological replicates were averaged and are presented as the relative quantity $(\mathrm{Rq})$ compared to act-2. Synchronized L1 larvae were treated with $35 \mu \mathrm{l}$ of auxin for $0,1,3$, and 6 hours, with a 6 -hour ethanol treatment used as a vehicle control. Error bars indicate standard error of the mean. Significance was calculated using a student's T test $\left({ }^{*} p<0.05^{* *} p<0.01\right.$ experimental samples compared to 0 -hour auxin treatment, $\# p<0.05 \# \#<<0.01$ experimental samples compared to 6 -hour ethanol vehicle treatment). 


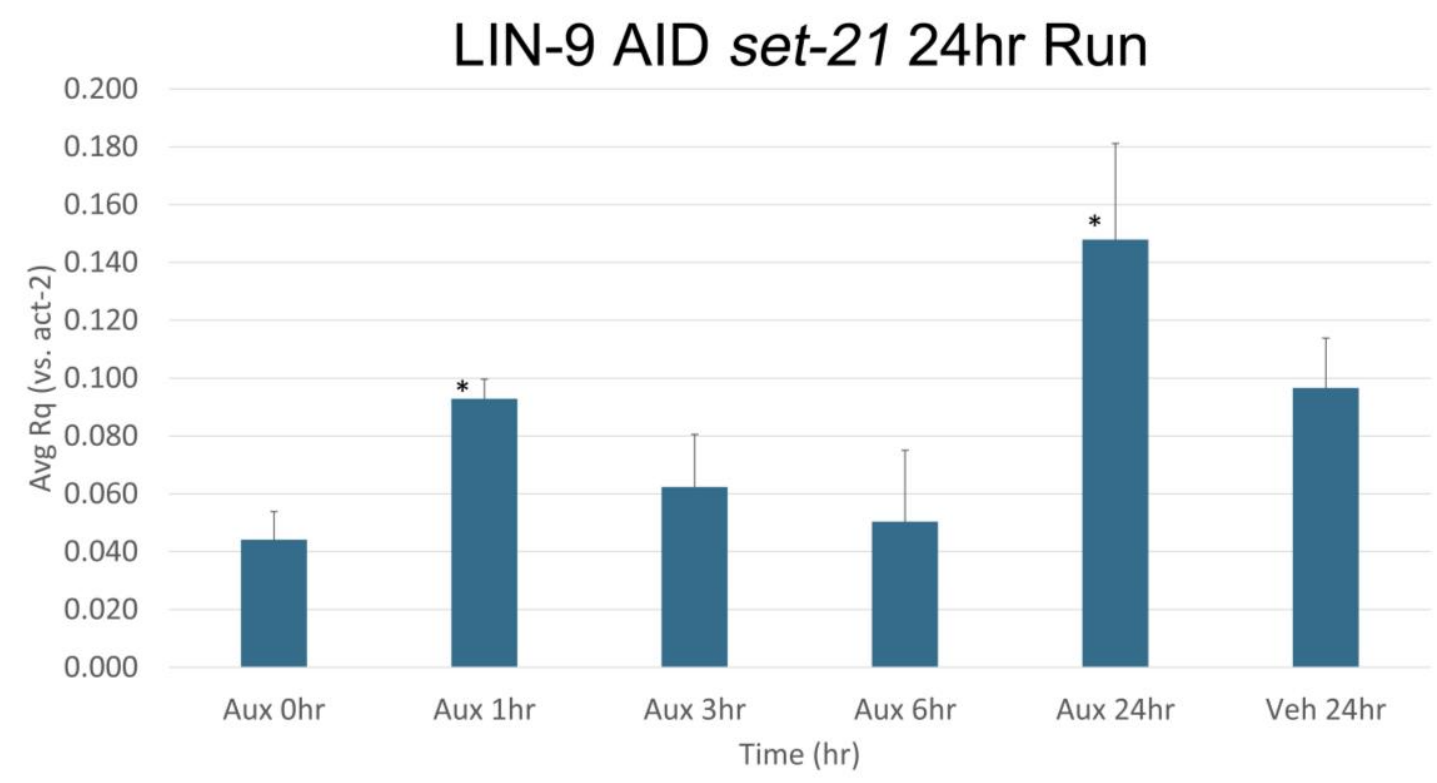

Figure 22 Average relative quantity $(\mathrm{Rq})$ of set-21 compared to act-2 over a 24-hour auxin time course in LIN-9 degron-tagged L1 larvae.

Expression values from 3 biological replicates were averaged and are presented as the relative quantity $(\mathrm{Rq})$ compared to act-2. Synchronized L1 larvae were treated with $35 \mu \mathrm{l}$ of auxin for $0,1,3$, and 6 hours, with a 6 -hour ethanol treatment used as a vehicle control. Error bars indicate standard error of the mean. Significance was calculated using a student's T test $\left({ }^{*} p<0.05{ }^{* *} p<0.01\right.$ experimental samples compared to 0 -hour auxin treatment, $\# p<0.05 \# \# p<0.01$ experimental samples compared to 6-hour ethanol vehicle treatment). 


\subsection{DISCUSSION}

Proper function of the DREAM, or DRM, transcriptional repressor complex is essential to maintain developmental control of the cell cycle. Removal of DREAM complex proteins causes a number of abnormal effects in $C$. elegans, including high temperature larval arrest and gene misexpression [6, 16, 22]. Mammalian MuvB, one of the three main components of DREAM, functions as both a transcriptional repressor and as an activator [5]. In C. elegans, MuvB thus far is only known to act as a transcriptional repressor [6]. It is currently unclear how MuvB mediates repression of DREAM target genes, thus an understanding of its individual proteins and their potential repressive roles is crucial to distinguishing MuvB's role in transcriptional repression.

The primary aim of this project was to establish how degradation of two MuvB proteins, LIN-9, the core protein of MuvB, and LIN-54, the sole DNA-binding protein of MuvB, would affect DREAM target gene expression [14, 23]. To accomplish this we used the AID system, a fasting-acting, efficient protein degradation system with minimal off-target effects [1, 2]. We assessed three known DREAM target genes, air-1, cdk-1, and set-21 following depletion of degron-tagged LIN-9 or LIN-54 in a 6-hour or 24-hour time course, with timepoints at 0-hour, 1-hour, 3-hour, 6-hour, and 24-hour. The establishment of a time course allowed us to infer when protein knockdown occurred and showed how expression levels rose with longer treatments. Following depletion of LIN-54, all our tested DREAM target genes became quickly upregulated as compared to our 0-hour 
auxin treatment control and 6-hour ethanol vehicle control. In contrast, depletion of LIN-9 had no effect on DREAM target gene expression.

We initially expected that both LIN-54 and LIN-9 degradation would result in the upregulation of DREAM target genes. LIN-9 is expected to be a core protein in the 5-subunit MuvB subcomplex, and as such we expected its degradation would result in significant disruption in MuvB activity [14]. In contrast, LIN-54 is the sole DNA-binding protein in MuvB. and as such we expected its degradation would result in MuvB from localizing to its gene targets and thus also impairing MuvB activity [23]. Our results suggest that MuvB DNA localization is critical to its function and that LIN-9 may not be fully required for the 5-subunit MuvB subcomplex to form.

Given our results, further study into the depletion of LIN-9 is a necessity. We would like to test how long and to what extent the LIN-9 protein depletion occurs. We would similarly test LIN-54 as well. It remains a possibility that LIN-9 knock-out is incomplete using the AID system as compared to LIN-54, which results in the observed discrepancy in our time course experiment. We strongly suggest further experimentation into the degradation of LIN-54 and especially LIN9 to determine their true importance to $C$. elegans MuvB and the DRM complex.

There were a few instances where the relative quantity of our 6-hour vehicle, that was treated with only ethanol, was higher than either one or both of our 0 -hour auxin treatment and our 1-hour auxin treatment. We believe this discrepancy may be attributed to stress more than ethanol having an effect on 
DREAM target gene expression. The source of this stress is more likely associated from the lack of nutrition and starvation the L1s underwent than the ethanol treatment.

Our results show that LIN-54, MuvB's sole DNA-binding protein is more important to the repression of DREAM target genes than LIN-9, the core protein of MuvB. Taking this a step further suggests that LIN-54 may be a critical component in DREAM complex-mediated transcriptional repression. To continue advancing our understanding of the DREAM complex and how its protein components function in transcriptional repression, additional experimentation should examine the effect auxin-induced degradation has on other components of the $C$. elegans DRM complex. 


\section{REFERENCES}

1. Lambrus, B. G., Moyer, T. C., \& Holland, A. J. (2018). Applying the auxininducible degradation system for rapid protein depletion in mammalian cells. Methods in cell biology, 144, 107-135. https://doi.org/10.1016/bs.mcb.2018.03.004.

2. Zhang, L., Ward, J. D., Cheng, Z., \& Dernburg, A. F. (2015). The auxininducible degradation (AID) system enables versatile conditional protein depletion in C. elegans. Development, 142(24), 4374-4384.

https://doi.org/10.1242/dev.129635.

3. Martinez, M., Kinney, B. A., Medwig-Kinney, T. N., Ashley, G., Ragle, J. M., Johnson, L., Aguilera, J., Hammell, C. M., Ward, J. D., \& Matus, D. Q. (2020). Rapid Degradation of Caenorhabditis elegans Proteins at Single-Cell Resolution with a Synthetic Auxin. G3, 10(1), 267-280.

https://doi.org/10.1534/g3.119.400781.

4. Holland, A. J., Fachinetti, D., Han, J. S., \& Cleveland, D. W. (2012). Inducible, reversible system for the rapid and complete degradation of proteins in mammalian cells. Proceedings of the National Academy of Sciences of the United States of America, 109(49), E3350-E3357.

https://doi.org/10.1073/pnas.1216880109.

5. Sadasivam, S., \& DeCaprio, J. A. (2013). The DREAM complex: master coordinator of cell cycle-dependent gene expression. Nature reviews. Cancer, 13(8), 585-595. https://doi.org/10.1038/nrc3556.

6. Goetsch, P. D., Garrigues, J. M., \& Strome, S. (2017). Loss of the Caenorhabditis elegans pocket protein LIN-35 reveals MuvB's innate function as the repressor of DREAM target genes. PLoS genetics, 13(11), e1007088. https://doi.org/10.1371/journal.pgen.1007088.

7. Pagano M. (1997). Cell cycle regulation by the ubiquitin pathway. FASEB journal : official publication of the Federation of American Societies for Experimental Biology, 11(13), 1067-1075. https://doi.org/10.1096/fasebj.11.13.9367342.

8. Jia, L., \& Sun, Y. (2009). RBX1/ROC1-SCF E3 ubiquitin ligase is required for mouse embryogenesis and cancer cell survival. Cell division, 4, 16. https://doi.org/10.1186/1747-1028-4-16. 
9. Ye, Y., \& Rape, M. (2009). Building ubiquitin chains: E2 enzymes at work. Nature reviews. Molecular cell biology, 10(11), 755-764.

https://doi.org/10.1038/nrm2780.

10. Nishimura, K., Fukagawa, T., Takisawa, H., Kakimoto, T., \& Kanemaki, M. (2009). An auxin-based degron system for the rapid depletion of proteins in nonplant cells. Nature methods, 6(12), 917-922.

https://doi.org/10.1038/nmeth.1401.

11. Kanke, M., Nishimura, K., Kanemaki, M., Kakimoto, T., Takahashi, T. S., Nakagawa, T., \& Masukata, H. (2011). Auxin-inducible protein depletion system in fission yeast. BMC cell biology, 12, 8. https://doi.org/10.1186/1471-2121-12-8.

12. Trost, M., Blattner, A. C., \& Lehner, C. F. (2016). Regulated protein depletion by the auxin-inducible degradation system in Drosophila melanogaster. Fly, 10(1), 35-46.

https://doi.org/10.1080/19336934.2016.1168552.

13. Sadasivam, S., Duan, S., \& DeCaprio, J. A. (2012). The MuvB complex sequentially recruits B-Myb and FoxM1 to promote mitotic gene expression. Genes \& development, 26(5), 474-489. https://doi.org/10.1101/gad.181933.111.

14. Fischer, M., \& Müller, G. A. (2017). Cell cycle transcription control:

DREAM/MuvB and RB-E2F complexes. Critical reviews in biochemistry and molecular biology, 52(6), 638-662.

https://doi.org/10.1080/10409238.2017.1360836.

15. Goetsch, P.D., Strome, S. 2019. DREAM Interrupted: Severing MuvB from DREAM's pocket protein in Caenorhabditis elegans impairs gene repression but not DREAM chromatin assembly. bioRxiv. https://doi.org/10.1101/671024.

16. Petrella, L. N., Wang, W., Spike, C. A., Rechtsteiner, A., Reinke, V., \& Strome, S. (2011). synMuv B proteins antagonize germline fate in the intestine and ensure C. elegans survival. Development, 138(6), 1069-1079. https://doi.org/10.1242/dev.059501

17. Patel, A. J., Wan, Y. W., Al-Ouran, R., Revelli, J. P., Cardenas, M. F., Oneissi, M., Xi, L., Jalali, A., Magnotti, J. F., Muzny, D. M., Doddapaneni, H., Sebastian, S., Heck, K. A., Goodman, J. C., Gopinath, S. P., Liu, Z., Rao, G., Plon, S. E., Yoshor, D., Wheeler, D. A., ... Klisch, T. J. (2019). Molecular profiling predicts meningioma recurrence and reveals loss of DREAM complex repression in aggressive tumors. Proceedings of the National Academy of Sciences of the 
United States of America, 116(43), 21715-21726.

https://doi.org/10.1073/pnas.1912858116.

18. Iness, A. N., Felthousen, J., Ananthapadmanabhan, V., Sesay, F., Saini, S., Guiley, K. Z., Rubin, S. M., Dozmorov, M., \& Litovchick, L. (2019). The cell cycle regulatory DREAM complex is disrupted by high expression of oncogenic BMyb. Oncogene, 38(7), 1080-1092. https://doi.org/10.1038/s41388-018-0490-y.

19. Forristal, C., Henley, S. A., MacDonald, J. I., Bush, J. R., Ort, C., Passos, D. T., Talluri, S., Ishak, C. A., Thwaites, M. J., Norley, C. J., Litovchick, L., DeCaprio, J. A., DiMattia, G., Holdsworth, D. W., Beier, F., \& Dick, F. A. (2014). Loss of the mammalian DREAM complex deregulates chondrocyte proliferation. Molecular and cellular biology, 34(12), 2221-2234.

https://doi.org/10.1128/MCB.01523-13.

20. Reichert, N., Wurster, S., Ulrich, T., Schmitt, K., Hauser, S., Probst, L., Götz, R., Ceteci, F., Moll, R., Rapp, U., \& Gaubatz, S. (2010). Lin9, a subunit of the mammalian DREAM complex, is essential for embryonic development, for survival of adult mice, and for tumor suppression. Molecular and cellular biology, 30(12), 2896-2908. https://doi.org/10.1128/MCB.00028-10.

21. Esterlechner, J., Reichert, N., Iltzsche, F., Krause, M., Finkernagel, F., \& Gaubatz, S. (2013). LIN9, a subunit of the DREAM complex, regulates mitotic gene expression and proliferation of embryonic stem cells. PloS one, 8(5), e62882. https://doi.org/10.1371/journal.pone.0062882.

22. Costello, M. E., \& Petrella, L. N. (2019). C. elegans synMuv B proteins regulate spatial and temporal chromatin compaction during development. Development, 146(19), dev174383.

https://doi.org/10.1242/dev.174383.

23. Tabuchi, T. M., Deplancke, B., Osato, N., Zhu, L. J., Barrasa, M. I., Harrison, M. M., Horvitz, H. R., Walhout, A. J., \& Hagstrom, K. A. (2011). Chromosomebiased binding and gene regulation by the Caenorhabditis elegans DRM complex. PLoS genetics, 7(5), e1002074. https://doi.org/10.1371/journal.pgen.1002074. 